\title{
Onset criteria for freely decaying isotropic turbulence
}

\author{
S. R. Yoffe ${ }^{1, *}$ and W. D. McComb ${ }^{2, \dagger}$ \\ ${ }^{1}$ Department of Physics, SUPA $\&$ University of Strathclyde, Glasgow G4 ONG, United Kingdom \\ ${ }^{2}$ SUPA School of Physics and Astronomy, University of Edinburgh, Edinburgh EH9 3JZ, United Kingdom
}

From direct numerical simulation (DNS) of turbulence decaying from specified initial conditions for the range of initial Taylor-Reynolds numbers $2.58 \leqslant R_{\lambda}(0) \leqslant 358.6$, it was found that the shape of the iconic curve of dimensionless dissipation versus Reynolds number depended strongly on the choice of measurement time. For our preferred time, a composite based on peak values in the dissipation and inertial transfer curves, the result was virtually identical to the forced, stationary case. In order to try varying the initial conditions, an additional run was performed, using the data from a stationary, forced simulation with $R_{\lambda}=335$ for the initial condition. The results of this suggested that the time taken for energy to pass through the cascade was about one half of an initial eddy turnover time. In the course of studying onset criteria, we found that the exponent for the power-law decay of the energy decreased with increasing Reynolds number and lay in the range $1.35 \leqslant n \leqslant 2.60$.

\section{INTRODUCTION}

The present paper is the culmination of an investigation into the dependence of the dimensionless dissipation rate $C_{\varepsilon}$ on Reynolds number in isotropic turbulence. Our approach over the last decade has been based on analysis of the real-space energy balance equation (the Karman-Howarth equation or KHE for short), with direct numerical simulations providing validation and the evaluation of constants. Our first work in this area was the reinterpretation of the Taylor dissipation surrogate by McComb et al[1] as a surrogate for the inertial transfer, which becomes equal to the dissipation at sufficiently large Reynolds numbers, corresponding to the development of an inertial range. This work was for freely decaying turbulence.

It is worth pointing out that the supplemental material for this paper contained what was claimed to be an exact theoretical expression relating the dissipation to the Reynolds number. This relied on a number of physical arguments, adding up to a derivation that led to the form

$$
C_{\varepsilon}\left(t_{e}\right)=C_{\varepsilon, \infty}\left(t_{e}\right)+\frac{C^{\text {decay }}\left(t_{e}\right)}{R_{L}\left(t_{e}\right)},
$$

and this was readily fitted to our experimental results. Here $R_{L}$ is the Reynolds number based on the integral scale $L$ (defined as $L=\int_{0}^{\infty} f(r) d r=$ $(3 \pi / 4 E) \int d k E(k) / k$, where $f(r)$ is the longitudinal correlation function, $E(k)$ the energy spectrum, and $E$ the total energy) while $C^{\text {decay }}\left(t_{e}\right)$ is a coefficient derived from the second- and third-order structure functions and the differential coefficient of the secondorder structure function with respect to time. A key feature is the fact that we had to evaluate each term in this expression at some fiducial time $t=t_{e}$ during the decay.

Unfortunately this derivation was not easy to understand and, in order to make the theory more accessible, we resorted to an approximate method in which

\footnotetext{
* sam.yoffe@strath.ac.uk

$\dagger$ wdm@ph.ed.ac.uk
}

we introduced asymptotic expansions of the structure functions in powers of the inverted Reynolds number. This technique had previously been used by Lundgren [2] to derive the Kolmogorov two-thirds law. Logically the present paper should have been the first outcome of this research, but our new method emphasised the difficulties with time dependence in the decaying case. So we turned instead to the derivation of an asymptotic theory of the dissipation rate in forced turbulence, and its verification using DNS: see McComb et al[3]. The result was similar to that for the decaying case, but of course without the time dependences, thus:

$$
C_{\varepsilon}=C_{\varepsilon, \infty}+\frac{C}{R_{L}},
$$

where $C_{\varepsilon, \infty}$ depends on the third-order structure function, while $C$ depends on both the second- and thirdorder structure functions. In principal we could include higher-order terms: the second-order term in the asymptotic expansions was needed when the work was extended to free decay of homogeneous magnetohydrodynamic turbulence by Linkmann et al[4]. However, several numerical tests suggested that the dependence on the Reynolds number is as indicated in (2), thus lending support to the idea that this is actually an exact result.

The extension of our new theory to freely decaying isotropic turbulence was done later by McComb and Fairhurst [5] and leads to two principal results. These are:

A: That the time-derivative in the KHE which was neglected in the derivation of the Kolmogorov ' $4 / 5$ ' law $[6,7]$ cannot be so neglected solely on the basis of a restriction to certain scales or to large Reynolds numbers.

B: That the neglect of this term can be quantified by a comparison of the free decay and forced forms of the asymptotic dimensionless dissipation rate, provided only that we know the correct value $t=t_{e}$ to choose as a fiduciary time.

The importance of this is that Kolmogorov [6] introduced the concept of local stationarity (as an aspect 
of local isotropy) and that this was further described by Batchelor [8] as local equilibrium. These were presented as general properties; and are not quite the same as arguments that the time derivative may be neglected as an approximation on one set of scales in comparison to another. Yet in practice one suspects that the two concepts are often blurred. Accordingly, it is helpful to have the general concepts ruled out as exact, rigorous properties, But the need to quantify local stationarity as an approximation provides an additional motivation (were one needed) to establish to what extent the asymptotic dimensionless dissipation rate depends on the decay time when the measurement is made, and that is the subject of this paper.

The paper is organised into the following sections:

Section 2 provides a short review of the study of the dependence of the dimensionless dissipation rate on Reynolds number with particular emphasis on the topics that are most relevant to the present work.

Section 3 discusses the nature of the problem of deciding when a decaying simulation is fully evolved, in comparison with the problem of doing this for a forced simulation, and proposes various possible onset criteria.

Section 4 presents details of the DNS and also gives the results for various statistical quantities as a function of both elapsed time and Reynolds number in order that the quality of the simulations can be assessed. This allows an assessment of criteria for choosing an evolved time based on the behaviour of the dissipation rate, the inertial transfer rate and the skewness factor.

Section 5 proposes a composite criterion, based the evolution in time of both the dissipation and the inertial transfer rates.

Section 6 gives the effect of individual choices of evolved time on the measurements of the dissipation rate.

Section 7 discusses the results, and puts forward suggestions for future work.

Appendix A is a brief introduction to power-law decay and compares our results to the field in general

Appendix B explores the alternative determination of the energy exponent by measuring the Taylor microscale.

\section{THE DIMENSIONLESS DISSIPATION RATE}

In recent years, there has been great interest in the Reynolds number dependence of the dissipation rate in homogeneous, isotropic turbulence (HIT). Apart from its intrinsic fundamental signifance, it is a key factor in the free decay of a turbulent fluid. Much of this work has been based on Taylor's expression for the dissipation rate, thus:

$$
\varepsilon=C_{\varepsilon}\left(R_{L}\right) U^{3} / L,
$$

which was put forward in 1935 by Taylor [9] on the basis of dimensional arguments. Here $U$ is the rms velocity of the fluid and $L$ is the integral length scale.
Note that we have explicitly indicated the dependence of $C_{\varepsilon}$ on the Reynolds number. Nowadays it is more usual to rearrange this as

$$
C_{\varepsilon}\left(R_{L}\right)=\varepsilon L / U^{3},
$$

so that $C_{\varepsilon}\left(R_{L}\right)$ may be interpreted as the dimensionless dissipation rate, rather than the Taylor prefactor. As early as 1953, Batchelor [10] (in the first edition of this book) presented evidence to suggest that $C_{\varepsilon}$ tends to a constant $C_{\varepsilon, \infty}$ with increasing Reynolds number. Later Sreenivasan [11] established, from a survey of investigations into grid-generated turbulence, that $C_{\varepsilon}$ became constant for Taylor-Reynolds numbers greater than about 50. This independence of viscosity is sometimes referred to as the dissipation anomaly.

For a Newtonian fluid, the dissipation rate is formally defined in terms of the kinematic viscosity $\nu$, thus:

$$
\hat{\varepsilon}=\frac{\nu}{2}\left(\frac{\partial u_{i}}{\partial x_{j}}+\frac{\partial u_{j}}{\partial x_{i}}\right)^{2} .
$$

As $\mathbf{u}(\mathbf{x}, t)$ is a random variable with zero mean, it follows that $\hat{\varepsilon}$ is the instantaneous dissipation rate, and is also a random variable. For a turbulent flow we introduce the mean dissipation rate, as:

$$
\varepsilon=\left\langle\frac{\nu}{2}\left(\frac{\partial u_{i}}{\partial x_{j}}+\frac{\partial u_{j}}{\partial x_{i}}\right)^{2}\right\rangle,
$$

where the angle brackets $\langle\ldots\rangle$ denote the operation of taking an average. To avoid any possible confusion, it should be noted that we will use the mean dissipation rate, along with the other averaged quantities, in our description of fluid turbulence. Accordingly, the concept of intermittency, which is related to the behaviour of single realizations, and is just one aspect of the phase-dependent behaviour of the velocity field, has no relevance to our discussion here. Here we begin by reviewing the relevant literature and summarising recent work on the behaviour of the dimensionless dissipation as the Reynolds number is increased.

Sreenivasan [11] had concluded that results for square-mesh grids suggested that the dimensionless dissipation rate became independent of Reynolds number for $R_{\lambda}>50$. He noted that there was a marked variation at lower Reynolds numbers but found this unsurprising. Where the inertia forces could be neglected, one might expect a relationship of the form

$$
C_{\varepsilon}=15(\pi / 2)^{1 / 2} / R_{\lambda} .
$$

This arose from the well known expression $\varepsilon=$ $15 \nu U^{2} / \lambda^{2}$, taken in conjunction with the approximation $L / \lambda \simeq(\pi / 2)^{1 / 2}$. He also remarked upon the lack of clear evidence for other flow configurations, but speculated that the asymptotic value of the dimensionless dissipation could depend on the initial conditions.

In an update, in 1998, Sreenivasan [12] presented data from four investigations using DNS. These were 
by Jiménez, Wray, Saffman and Rogallo [13], Yeung and Zhou [14] and Cao, Chen and Doolen [15], all studying forced turbulence, and by Wang, Chen, Brasseur and Wyngaard [16], who studied both decaying and forced turbulence. He concluded that $C_{\varepsilon, \infty} \sim O(1)$, with $C_{\varepsilon, \infty}$ sensitive to both the initial conditions and the method of forcing. Wang et al [16] found $C_{\varepsilon, \infty} \sim 0.62$ (decay) and $0.42-0.49$ (forced).

In considering the scatter of results, Sreenivasan highlighted the relevance of large-scale resolution, noting that in the forced simulations the integral scale and the box size were about the same. More recently, Burattini, Lavoie and Antonia [17] presented an experimental investigation of $C_{\varepsilon}$ for several different experimental setups including grid turbulence (both active and passive grids) and a variety of bluff body wakes. Their figures 1 and 2 summarize their results, which show a spread of $C_{\varepsilon, \infty}$ values from $0.5-2.5$ for $R_{\lambda}>50$. These authors drew attention to the difficulties of measuring the dissipation rate, remarking that grid turbulence has the advantage that it can be determined from the decay rate. They noted that the asymptotic value of the dimensionless dissipation not only varied from one flow type to another, but also could depend on details of the body producing the turbulence.

However, for forced DNS at least, there seemed to be a general trend to an asymptotic value of slightly less than 0.5. In an adaptation of the figure in [12], Burattini et al[17] presented additional results for forced DNS from Gotoh, Fukuyama and Nakano [18] and from Kaneda, Ishihara, Yokokawa, Itakura and Uno [19]. Here we also present an adaptation of this figure, as Fig. 1, where we have added results for DNS of free decay from McComb, Berera, Salewski and Yoffe [1], along with some new results from the present investigation. For forced DNS we have also added results from Donzis, Sreenivasan and Yeung [20].

Measurements of $C_{\varepsilon}$ were made by Pearson, Krogstad and van der Water [21] in various shear flows (including several forms of grid-generated turbulence). From our present point of view, their conclusion that for $R_{\lambda} \geqslant 300$ a value of $C_{\varepsilon} \sim 0.5$ seemed to be a good universal approximation for flow regions free of strong mean shear, is of particular interest. Later Pearson and co-workers [22] used DNS to study stationary HIT, up to a Taylor-Reynolds number of about 220. They found that the dimensionless dissipation rate slowly approached a value of $C_{\varepsilon} \sim 0.5$, although we should note that their simulation was slightly compressible.

A rather more extensive investigation than most was carried out by Bos, Shao and Bertoglio [23], who used a variety of methods to study both decaying and forced turbulence. Pseudospectral DNSs were carried out on $64^{3}-256^{3}$ lattices, with a maximum value of $R_{\lambda} \sim 100$ for their forced runs. They also used largeeddy simulation (LES) and the well-known single-time model closure EDQNM, with $R_{\lambda}$ up to $\sim 2000$.

For the simulations of free decay, they tried four different initial spectra and concluded that, after a short

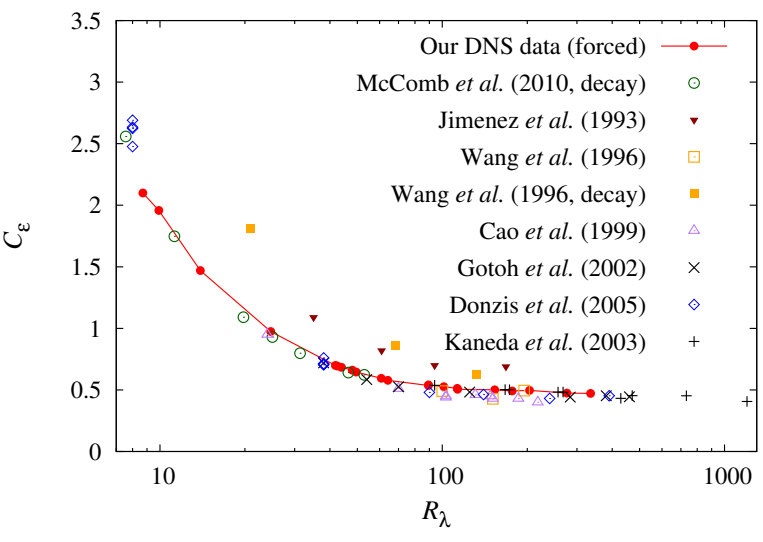

FIG. 1. A review of the data obtained for $C_{\varepsilon}$ as a function of Taylor-Reynolds number, $R_{\lambda}$, from numerical simulations. Data sets from decaying turbulence are indicated in the figure legend. Along with data obtained the present work, and from McComb et al [1], we also plot results from Jiménez et al [13], Wang et al [16], Cao et al [15], Gotoh et al [18], Donzis et al [20] and Kaneda et al [19].

transient, the different simulations all led to the same value of the dimensionless dissipation. In the case of the forced simulations, they tried three different forcing schemes, and found that all three ended up with values of $C_{\varepsilon}$ which oscillated about a constant value of about $C_{\varepsilon}=0.6$. Again the only difference was in the initial transient and, in this case, the size of the oscillations about the mean value.

The techniques of LES and EDQNM were used to extend the investigation to much higher Reynolds numbers. The clear result was a significant difference between the decaying and forced cases. As mentioned above they found $C_{\varepsilon, \infty} \sim 0.6$ for the forced case while for free decay the asymptotic dimensionless dissipation took the much larger value of $C_{\varepsilon, \infty} \sim 1$.

Bos et al discussed their results in the context of other work. At low Reynolds numbers they found that their results tended to the form $a R_{L}^{-1}$, as predicted by Sreenivasan (see above), with $a \sim 20$. They noted reasonable agreement with the result of Mydlarski and Warhaft [24], who found $C_{\varepsilon, \infty} \sim 0.9$ for grid-generated turbulence, but remarked upon the disagreement with Pearson et al [21], who obtained a much lower value, and speculated about the difficulty of measuring the integral scale and also the possibility of finite-size effects. A particularly interesting feature of this work is their conclusion on phenomenological grounds that the value of $C_{\varepsilon, \infty}$ should be larger for free decay than for forced HIT. We shall return to this later in the context of our own work in the present paper. At this point we mention our earlier work on the dimensionless dissipation in freely decaying turbulence.

McComb, Berera, Salewski and Yoffe [1] made a numerical investigation of energy dissipation and transfer rates in decaying isotropic turbulence with $R_{\lambda}\left(t_{e}\right)<60$. They tried three different initial spectra, with both $k^{2}$ and $k^{4}$ low-wavenumber behaviour, 
and measured the dimensionless dissipation rate $C_{\varepsilon}$ using the time at which the peak of the dissipation rate occurred as their measurement time. The results were comparable to forced simulations, with $C_{\varepsilon, \infty} \sim 0.5$. They also compared the dissipation rate with the transfer rate and the dissipation surrogate $U^{3} / L$ for each of the three initial spectra, and showed that $U^{3} / L$ was a better surrogate for transfer than the dissipation rate. A plot of the ratio $\varepsilon / \varepsilon_{T}$ (again, measured at the peak of the dissipation rate, $t_{e}$ ) for the three spectra, showed the approach to $\varepsilon_{T}=\varepsilon-\Delta$, where $\Delta$ is due to the finite cascade time and decaying nature of the turbulence.

Since then we have developed theoretical arguments to support our conclusion that Taylor's expression is actually a surrogate for the inertial transfer rate (rather than for the dissipation), and have found that results from the present DNS support this view. We do not claim that this interpretation is entirely new: some commentators seem to have taken the same view (for instance, see the books by Batchelor [10], Tennekes and Lumley [25], Davidson [26] and Sagaut and Cambon [27]). However, it certainly runs counter to the view which underpins many investigations in laboratory and environmental flows, along with more recent work on DNS.

We should also mention the comprehensive study of decaying turbulence by Vassilicos and co-workers [28][31], who used a variety of fractal grids, active grids and regular grids. This work underlines the need to understand better what is truly universal in turbulence. Their various values for $C_{\varepsilon, \infty}$ are given, along with those of others, in Table I. Note that for fractal grids they found that the dimensionless dissipation rate does not seem to have a finite asymptotic value as the Reynolds number tends to infinity, whereas for regular grids they favour a value of $C_{\varepsilon, \infty}$ about unity. In contrast, Krogstad and Davidson [32] found that $C_{\varepsilon} \sim 0.6$ for both multiscale and conventional grids at a Taylor-Reynolds number of about 70 . It should be noted that these authors plot (in effect) $3 C_{\varepsilon} / 2$ against downstream distance in their figure 11.

Lastly, for completeness we note the investigation of the relationship between self-preservation and the dependence of the dissipation rate on Reynolds number in two kinds of decaying turbulence by Djenidi et $a l[33]$, as the most recent work of this group.

\section{THE NATURE OF THE PROBLEM}

Free decay of isotropic turbulence is the principal benchmark problem for both phenomenological and theoretical treatments of the turbulence problem. Essentially it is believed that the turbulence kinetic energy decays as a power law, that is: $U^{2} \sim t^{-n}$, where the exponent $n$ has to be determined. But, more than seven decades after the study of this problem began, there is an absence of agreement or even much consensus on the value of this exponent.

When formulated as a problem in mathematical physics, and realized in practice in a direct numerical simulation (DNS), it is an initial value problem where the initial velocity field is arbitrarily chosen to have a Gaussian distribution and a specified form of energy spectrum. As the system evolves in time (or is iterated forward in time), one expects to achieve a velocity field which is determined by the Navier-Stokes equations. In this respect it is like the problem of forced isotropic turbulence. But in other respects it is not. In principle (and in practice) we can run a forced simulation forward in time until we are sure that we are observing Navier-Stokes turbulence. The end state is stationary turbulence which fluctuates about a fixed mean value of the energy, with the fluctuating mean dissipation rate lagging behind, reflecting the time needed for energy to pass through the cascade [35]. It should perhaps be emphasised that these are not turbulent fluctuations, but are analogous to the fluctuations in total energy of the canonical ensemble of statistical physics. As in that case, the relative magnitude of the fluctuations should decrease as the system size is increased.

However, in the case of free decay, the energy is already decaying, even before turbulence has been established. This raises a question which, as far as we know, has not been formally addressed: at what time in the decay process, $t=t_{e}$ (say), can the turbulence be said to be fully evolved? And what exactly do we mean by 'fully evolved'?

Inevitably the situation is complicated by the historical fact that the problem has been mostly studied experimentally in the form of grid-generated or grid turbulence. Thus current assessments tend to consist of a mix of results from laboratory experiments and increasingly from DNS (i.e. numerical experiments). Hence, the problem in making comparisons - that is, of 'like with like' — lies in the detailed specification of the initial conditions.

Since the late 1950s, the formulation of the theoretical problem has been based on an analogy with statistical physics. The initial field is taken to be Gaussian and to have a spectrum which is peaked near the origin. Development with time then involves the nonlinear coupling process transferring energy to ever higher wavenumbers, until the process is limited by viscosity. Thus, providing only that the initial spectrum satisfies some quite weak conditions, the resulting turbulence should be universal. The extension to DNS can and should follow this route, to the extent permitted by the restrictions imposed by the computational process.

In the case of grid turbulence, the situation is very much more complicated. To begin with, strictly it involves free decay of a stationary flow in space rather than time, so that a Galilean transformation is required to interpret the problem as one of time dependence. Then, there can be many choices of initial conditions, depending on the type and solidity of grid. There can also be other complicating factors to do with the design of the wind tunnel. In short we may have little idea of whether any given grid turbulence experiment satisfies the requirements of the theoretical/computational formulation. Hence it behoves us 


\begin{tabular}{|c|c|c|c|}
\hline Reference & $C_{\varepsilon, \infty}$ & $R_{\lambda}$ & Comments \\
\hline$\overline{\overline{\text { Sreenivasan }[11]}}$ & 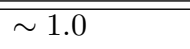 & 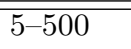 & 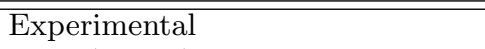 \\
\hline Jiménez et al [13] & $\sim 0.65$ & $35-168$ & DNS (forced) \\
\hline \multirow[t]{2}{*}{ Wang et al $[16]$} & $\sim 0.42-0.49$ & $100-195$ & DNS (forced) \\
\hline & $\sim 0.62$ & $21-132$ & DNS (decay) \\
\hline Yeung and Zhou $[14]$ & $\sim 0.4$ & $38-240$ & DNS (forced) \\
\hline \multirow[t]{2}{*}{ Sreenivasan [12] } & $\sim 0.5$ & $20-250$ & DNS from [14-16] (forced) \\
\hline & $\sim 0.78$ & $35-195$ & DNS from $[13,16]$ (forced, decay) \\
\hline Cao et al $[15]$ & $0.39-0.45$ & $24-218$ & DNS (forced) \\
\hline Pearson et al [21] & $0.4-0.6$ & $50-1200$ & Experimental \\
\hline Gotoh et al [18] & $\sim 0.5$ & $38-460$ & DNS (forced) \\
\hline Kaneda et al [19] & $0.4 \sim 0.5$ & $94-1201$ & DNS (forced) \\
\hline Pearson et al $[22]$ & $\simeq 0.5$ & $50-1200$ & DNS (forced), compressible \\
\hline Donzis et al [20] & $\sim 0.5$ & $10-400$ & DNS (forced) \\
\hline Burattini et al [17] & $0.5-2.5$ & $50-1100$ & Experimental \\
\hline \multirow[t]{2}{*}{ Bos et al $[23]$} & $\sim 0.6$ & $0-2000$ & DNS, LES and EDQNM (forced) \\
\hline & $\sim 1$ & $0-2000$ & DNS, LES and EDQNM (decay) \\
\hline McComb et al [1] & $\sim 0.5$ & $3-60$ & DNS (decay) \\
\hline Krogstad and Davidson [34] & $\sim 1$ & & Experimental (regular grid) \\
\hline Valente and Vassilicos [30] & $\sim 0.5$ & $100-570$ & Experimental (various grids) \\
\hline Valente and Vassilicos [31] & $0.9-1.0$ & & Experimental (regular grid) \\
\hline
\end{tabular}

TABLE I. Some representative values for the asymptotic dimensional dissipation rate $C_{\varepsilon, \infty}$ from the literature. Bos et al [23] obtained $R_{\lambda} \leqslant 2000$ using LES, and $R_{\lambda} \leqslant 100$ using DNS.

to be cautious about drawing parallels between DNS and any particular laboratory experiment. Indeed, the need to reconcile DNS simulations with grid turbulence is a developing field of research: see the recent work by Meldi and Sagaut [36], which assesses the effect of perturbations on the energy spectrum.

At the same time, it is not surprising that the field should be so controversial and confused. But, while we do not attempt to offer any remedy for that here, we do think that it emphasises the need to have a clear understanding of at least the theoretical/numerical formulation and assisting in this is our purpose in the present paper.

\section{A. Possible criteria for an evolved decay time $t=t_{e}$}

Naturally if one is concerned with measuring the exponent $n$ in the power law for the decay of the total energy, then the onset criterion should be based on the occurrence of power-law behaviour. Even then there are two ways of doing this. We could use a log-log plot of energy against decay time, or we can rely on the well known result that the Taylor microscale $\lambda$ is proportional to the square root of the decay time when the decay follows a power law, irrespective of the value of the exponent (see, for example, Section 7.5.1 of reference [37]. This criterion has also been used in the context of measurements of $C_{\varepsilon}$ by Wang et $a l[16]$ and Bos et al [23].

However, significant variation in the dimensionless dissipation rate occurs at very low Taylor-Reynolds numbers and this raises the possiblity that our choice of evolution time $t_{e}$ should be based in some way on the evolution of the dissipation rate, as was done by Fukayama et al [38] and by McComb et al[1]. Also, ideally we would like the time $t_{e}$ to be as small as possible, since the system is decaying; and the longer we wait the smaller the time-series that we have to work with.

There are at least four possible candidates for the choice of a criterion to determine the evolved time. These are as follows:

1. The onset of power-law decay of the total turbulence energy;

2. The onset of $t^{1 / 2}$ scaling of the Taylor microscale $\lambda$;

3. The occurrence of peak dissipation and/or transfer rate;

4. The occurrence of a peak in the skewness of the longitudinal velocity derivative when plotted against time.

It should be noted that the first two criteria are essentially variants of the same thing. Nevertheless, we shall treat each of these four potential criteria separately.

\section{B. Development of nonlinear energy transfer}

When considering whether the flow is fully developed, we know that the fluid motion is initially random but not turbulent. The main characteristic of turbulence is the growth of inertial transfer of kinetic energy by means of nonlinear coupling. Let us denote the inertial/nonlinear transfer rate by

$$
\varepsilon_{T}(t)=\max \Pi(k, t), \quad \Pi(k, t)=\int_{k}^{\infty} d k T(k, t),
$$


where $\Pi(k, t)$ is the transport power spectrum and $T(k, t)$ the transfer spectrum which may be expressed in terms of the triple moment, in the usual way [37]. Hence $\varepsilon_{T}$ represents the maximum rate of energy transfer through wavenumbers.

It is instructive to make a comparison between decaying and forced turbulence. We begin with the latter.

\section{Forced turbulence:}

1. At low Reynolds numbers, energy is also dissipated from low wavenumbers, so not all energy passes through the cascade. Therefore, we may expect to find $\varepsilon_{T}<\varepsilon$.

2. At higher Reynolds numbers, dissipation from low wavenumbers becomes negligible, and all energy dissipated must travel through the cascade. In this case we expect to find $\varepsilon_{T}=\varepsilon$.

Also, since the flow is forced and in a steady state, we must have the input $\varepsilon_{W}=\varepsilon$.

\section{Decaying turbulence:}

1. Even at high Reynolds numbers, we expect that $\varepsilon_{T}(t)=\varepsilon(t+\Delta t)<\varepsilon(t)$, where the equality implies that energy transferred at time $t$ will be dissipated at a later time, $t+\Delta t$, and the inequality is due to the fact that the system is decaying.

2. McComb et al[1] showed that $U^{3} / L$ is a better surrogate for $\varepsilon_{T}$ than $\varepsilon$. In this case,

$$
C_{\varepsilon}=\frac{\varepsilon L}{U^{3}} \sim \frac{\varepsilon}{\varepsilon_{T}},
$$

and the approach of $C_{\varepsilon}$ to a plateau can be seen as the development of an inertial range.

3. For the case of decaying turbulence, $\varepsilon(t)>$ $\varepsilon_{T}(t)$ and so the measured plateau will satisfy $C_{\varepsilon, \infty}^{\text {decay }} \geqslant C_{\varepsilon, \infty}^{\text {forced }}$. See also the recent work on this particular point by $\mathrm{McComb}$ and Fairhurst [5].

If one starts with energy concentrated in the low wavenumbers; then, during the transition period, when the turbulence is developing, one can measure $\varepsilon_{T}(t)>\varepsilon(t)$ as the energy is redistributed among modes. This is an indication that the turbulence is not fully developed. It is important that the measurement time $t=t_{e}$ should be taken late enough in the decay in order to ensure that turbulence has been properly established, but early enough for larger Reynolds numbers to be explored.

\section{NUMERICAL SIMULATIONS}

We used a pseudospectral DNS, with full dealiasing performed by truncation of the velocity field according

\begin{tabular}{r|lll|l|ll}
$N$ & $\nu_{0}$ & $R_{L}(0)$ & $R_{\lambda}(0)$ & $t_{\max }$ & $R_{L}\left(t_{\max }\right)$ & $R_{\lambda}\left(t_{\max }\right)$ \\
\hline \hline 128 & 0.1 & 3.236 & 2.582 & $12.9 \tau(0)$ & 0.1702 & 0.1512 \\
128 & 0.07 & 4.623 & 3.688 & $12.9 \tau(0)$ & 0.3861 & 0.3217 \\
128 & 0.05 & 6.473 & 5.164 & $12.9 \tau(0)$ & 0.7795 & 0.6209 \\
128 & 0.03 & 10.79 & 8.606 & $12.9 \tau(0)$ & 2.132 & 1.615 \\
128 & 0.02 & 16.18 & 12.91 & $12.9 \tau(0)$ & 4.284 & 3.048 \\
128 & 0.01 & 32.36 & 25.82 & $64.4 \tau(0)$ & 4.477 & 3.705 \\
128 & 0.007 & 46.23 & 36.88 & $64.4 \tau(0)$ & 6.920 & 5.303 \\
128 & 0.005 & 64.73 & 51.64 & $64.4 \tau(0)$ & 9.679 & 6.845 \\
256 & 0.0025 & 129.5 & 103.3 & $64.4 \tau(0)$ & 17.55 & 10.65 \\
256 & 0.0018 & 179.8 & 143.4 & $64.4 \tau(0)$ & 22.76 & 12.92 \\
512 & 0.00072 & 449.5 & 358.6 & $51.7 \tau(0)$ & 63.76 & 26.42 \\
\hline 1024 & 0.0002 & 3828.2 & 353.7 & $3.19 \tau(0)$ & 1742.2 & 182.9
\end{tabular}

TABLE II. Summary of the simulations and their initial parameters. With the exception of the $1024^{3}$ run, all runs started from a Gaussian random-field with an initial energy spectrum which goes as $k^{4}$ at low $k$ and an ensemble of 10 was created by using different seeds to the initial field generation. The $1024^{3}$ run instead used an ensemble of 5 simulations which started from evolved fields obtained from a stationary simulation. The total simulation time is given by $t_{\max }$ in terms of the initial value of the large-eddy turnover time $\tau(0)=L(0) / U(0)$.

to the two-thirds rule. Time advancement for the viscous term was performed exactly using an integrating factor, while the nonlinear term used Heun's method (second-order predictor-corrector). In general, each run was started from a Gaussian-distributed random field with an initial energy spectrum (which behaves as $k^{4}$ for the low- $k$ modes),

$$
E(k, 0)=c\left(k / k_{0}\right)^{4} \exp \left[-\left(k / k_{0}\right)^{2}\right],
$$

with $c=0.266$ and $k_{0}=3.536$. The one exception was a simulation started from an evolved $1024^{3}$ field.

For each Reynolds number studied, the only initial condition changed was the value assigned to the (kinematic) viscosity, $\nu_{0}$. An ensemble was generated by starting the simulations from different random initial field configurations (which all have the same $E(k, 0)$ defined above). This ensemble, together with shell averaging, was used to calculate statistics. The ensemble size for each Reynolds number discussed in this work was 10 , with the exception of decay from an evolved $1024^{3}$ field, for which only an ensemble of five realisations could be obtained. Simulations were run using lattices of size $64^{3}, 128^{3}, 256^{3}, 512^{3}$ and $1024^{3}$, with corresponding initial Reynolds numbers ranging from $R_{\lambda}(0)=2.58$ up to 358.6. Since the simulations are decaying, measurements are made at progressively lower Reynolds number. The smallest resolved wavenumber was $k_{\min }=2 \pi / L_{\text {box }}=1$ in all simulations, while the maximum wavenumber always satisfied $k_{\max } \eta>1.0$, where $\eta$ is the Kolmogorov dissipation lengthscale[39]. The integral scale, $L$, was found to lie between $0.35 L_{\text {box }}$ for our lowest Reynolds number and $0.18 L_{\text {box }}$ for our largest. Spectral quantities have been shell averaged using the smallest spacefilling shell width, $\Delta k=1$. A summary of these simulations is given in table II.

Our numerical code has been benchmarked using es- 
tablished test problems such as the Taylor-Green vortex [40] and our results are in agreement with those of Brachet et al[41]. Comparison has also been made to data obtained using the freely-available pseudospectral code hit3d[42], with good agreement being found. Since an important constraint of this work is the condition of isotropy, we have verified that the isotropy spectra measured for the DNS steady-state ensembles do not exhibit significant deviation from an isotropic system. This was done by comparing the average energy in the directions of two randomly-orientated unit vectors for each wavevector. Full details of benchmarking, statistics and the error analysis will be found in $[3,43]$ and in the thesis by Yoffe [44]. In addition, energy and transfer spectra for some of these simulations, as well as integral parameters, may be found in Section 4.1, pages 102-105 of the same thesis. We note also that simulation data from this investigation is available to download.

\section{A. Resolution of the integral scales during the simulations}

Once a simulation is started, the total energy simply begins to decay. But energy spectra show a more complicated behaviour and evolve from the initial chosen shape as the energy spreads out to increasing wavenumber. Similarly the transfer spectrum evolves from an initial value of zero. These facts are well known and we do not report such results in detail here. Instead, we concentrate on those results which are most relevant to the rest of the paper.

In view of the growing recognition of the importance of having the integral length scale fully resolved, we show the time evolution of the integral length scale during the decay in Fig. 2. The integral length scale, $L$, increases during decay, as the energy becomes concentrated at larger and larger length scales. We wish to check that the integral scale remains resolved during the simulation; that is $L<L_{\text {box }}=2 \pi$. The figure shows the variation of $L / L_{\mathrm{box}}$ with time for a range of initial Reynolds numbers. The ratio becomes smaller (i.e. better) as Reynolds number is increased.

\section{B. The occurrence of peak dissipation rate (at $\left.t=t_{\varepsilon}\right)$ and transfer rate (at $\left.t=t_{\Pi}\right)$}

The existence of a peak in the variation of any quantity with time during the decay offers the possibility of a well-defined criterion, which would allow the results of one investigation to be compared with those of another. As seen in Fig. 3, in those of our simulations with $R_{\lambda}(0)>25$, the dissipation rate initially increases and develops a peak. This peak value is easily identified, so we denote the corresponding time to the peak by $t=t_{\varepsilon}$. As mentioned earlier, this time $t_{\varepsilon}$ was the evolution criterion used for decaying simulations by Fukayama et al [38].

However, for $R_{\lambda}(0) \lesssim 25$, dissipation dominates from the start and there is no peak in $\varepsilon$. Instead, con-

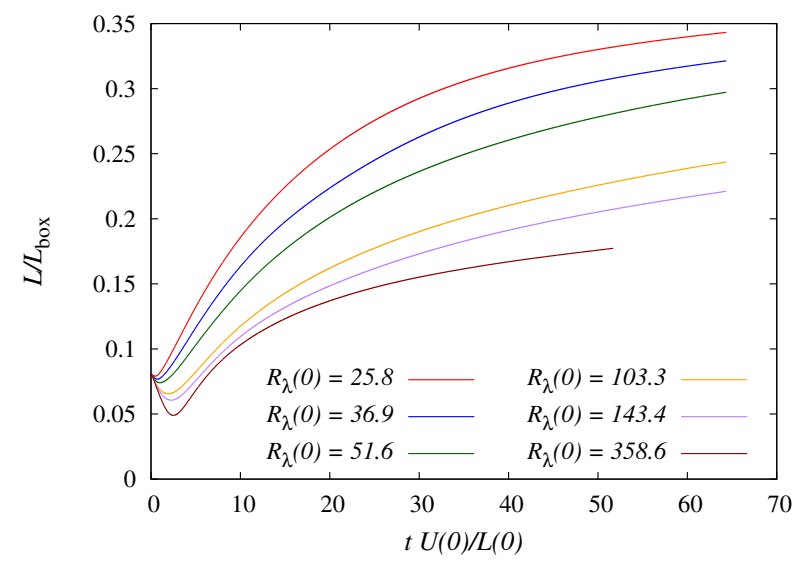

FIG. 2. The variation of the ratio of the integral scale to the box size as time progresses.

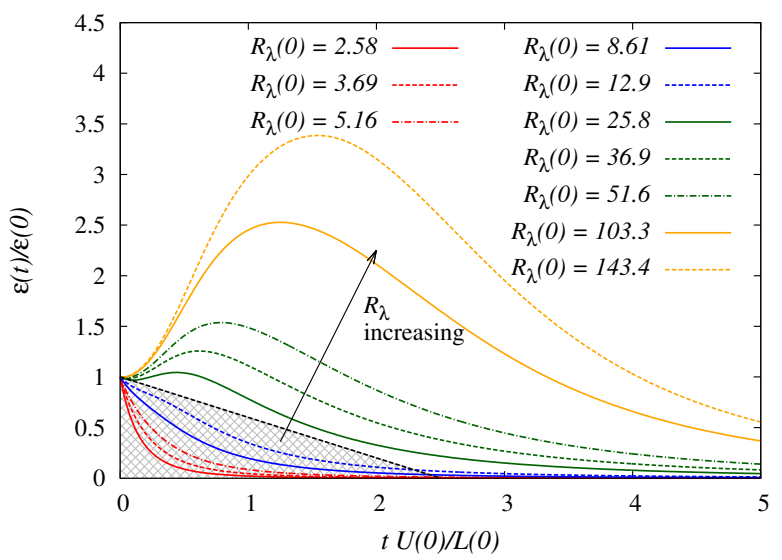

FIG. 3. Evolution of the dissipation rate $\varepsilon$ for a range of simulations. For the lowest Reynolds numbers, as highlighted by the grey shading, there is no initial increase.

sider time series for the maximum inertial flux, $\varepsilon_{T}$, as shown in Figure 4. Here we see that all the curves have peaks, and accordingly we may introduce a time $t=t_{\Pi}$ as the position of the peak in the inertial flux.

\section{The occurrence of the peak skewness: at $t=t_{S}$}

The velocity derivative skewness is often used as a criterion for fully developed flow. Developed turbulence has a non-Gaussian probability distribution. In a simulation the skewness starts from $S(0)=0$, since the initial field is Gaussian, and grows with time as the nonlinear interactions develop. We show our results for the skewness in Figure 5 as a function of time for various Reynolds numbers. It may be seen that the skewness asymptotes towards a value of $S \sim-0.5$ as both time and the Reynolds number increase. This is in broad agreement with the results of other investigations as the skewness has been found to be $S \sim-0.5$ for stationary turbulence: see Ishihara et 


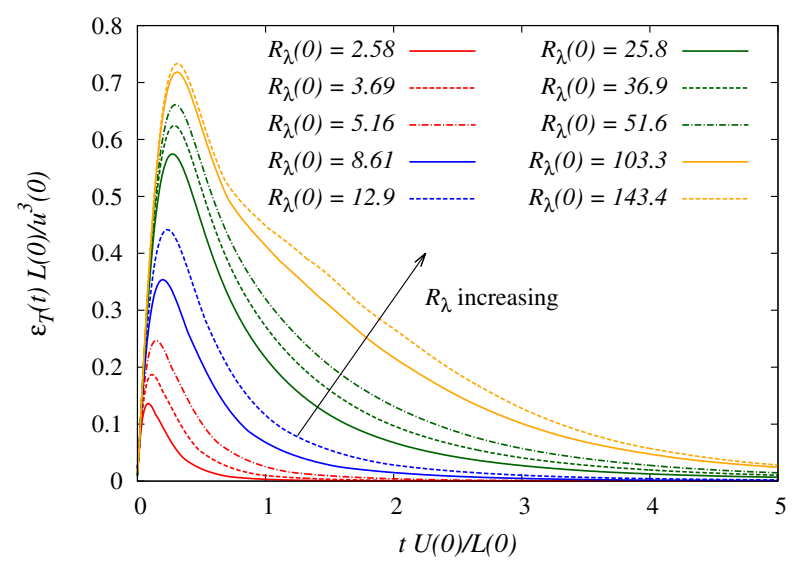

FIG. 4. Evolution of the maximum inertial flux $\varepsilon_{T}$ for a range of simulations.

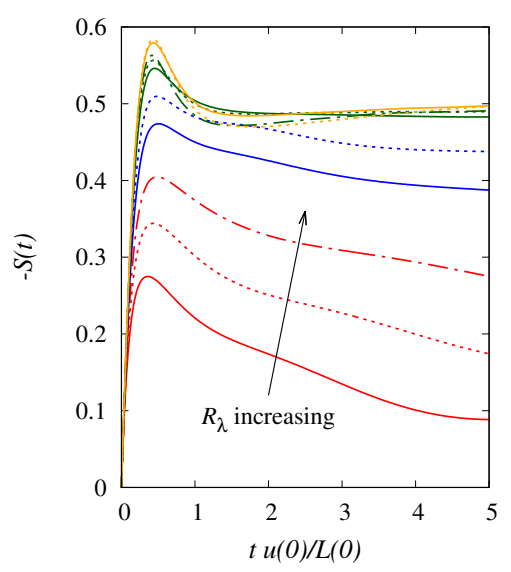

$$
\begin{aligned}
R_{\lambda}(0) & =2.58 \\
R_{\lambda}(0) & =3.69 \ldots \ldots . \\
R_{\lambda}(0) & =5.16 \ldots . \cdot \\
R_{\lambda}(0) & =8.61 \\
R_{\lambda}(0) & =12.9 \ldots \ldots . \\
R_{\lambda}(0) & =25.8 \\
R_{\lambda}(0) & =36.9 \ldots \ldots . \\
R_{\lambda}(0) & =51.6 \ldots . . \\
R_{\lambda}(0) & =103.3 \\
R_{\lambda}(0) & =143.4
\end{aligned}
$$

FIG. 5. Evolution of the velocity derivative skewness, $S(t)$. All Reynolds numbers display a peak, with higher Reynolds numbers also later developing plateaux for $t>$ $3 \tau(0)=3 L(0) / U(0)$.

al [45]; Machiels [46]; Vincent and Meneguzzi [47]; Kerr [48]; Gotoh et al [18]; Jiménez et al [13]; and Wang et al [16].

For large enough Reynolds numbers, a plateau develops around $S \sim-0.5$. The same set of Reynolds numbers which did not exhibit a peak in $\varepsilon$ are the ones which do not reach a plateau here. However, for all $R e$, there is a peak, corresponding to the evolved time $t_{S}$. Like $t_{\Pi}, t_{S}$ occurs very early in the decay for all Reynolds numbers.

\section{Decay from an evolved field}

In an experiment to provide a more realistic starting point for DNS of free decay, we used the data from a forced, stationary simulation to set the initial conditions. That is, we started from an evolved field which was taken from the $1024^{3}$ simulation with $R_{\lambda}=335$. As this data is, in effect, a solution to the forced Navier-Stokes equation, inevitably there is

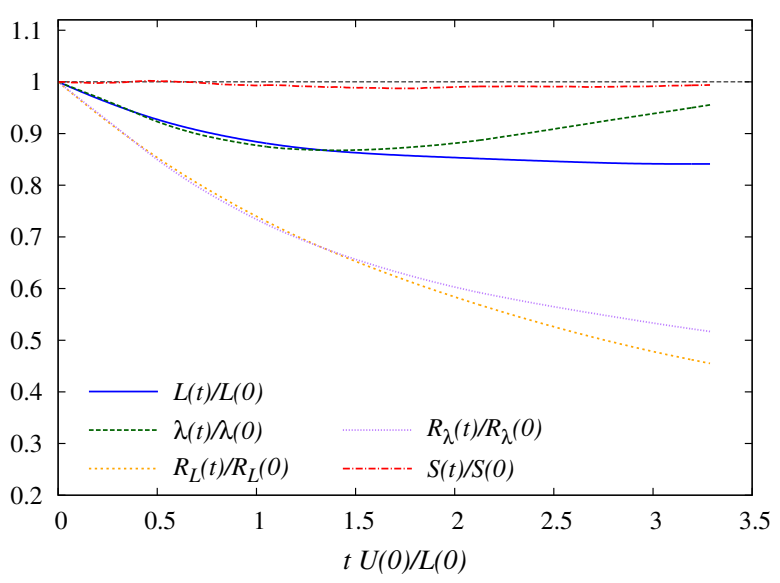

FIG. 6. Time evolution of length-scales, Reynolds numbers and skewness for decaying turbulence with an evolved initial condition.

a transient after the forcing is 'switched off' as the system adapts to the lack of an energy input.

This was a straightforward matter, with the only problems arising from the use of large data sets and the consequent long run times. We used an ensemble of five initial fields (separated by one large eddy turnover time, $\tau(0))$. It is of interest to note that $\tau(0)=$ $L / U=1.94 \mathrm{~s}$ from the stationary simulation which is much longer than initial turnover time for a Gaussian initial condition $\tau(0)=0.777 \mathrm{~s}$.

The evolution of various parameters with dimensionless decay time is shown in Figure 6. Evidently the normalised length scales and Reynolds numbers decay from $t=0$. The exception is the skewness (which had already reached a stationary value of $S=0.55$ ) which seems to adopt a slightly lower value, but does not vary significantly.

In Figure 7 we show the evolution of the dissipation and transfer rates. We note that the total energy and inertial flux, $\varepsilon_{T}$, start to decay straight away. But there is a period of about $\sim 0.5 \tau(0)$ during which the dissipation rate remains constant. This essentially measures the time it takes for energy to pass through the cascade, since during this time the dissipation from high wavenumbers is not 'aware' of the change which has occurred at low wavenumbers.

We also note that the dissipation rate appears to mimic the transfer rate. We tested this by shifting the $\varepsilon_{T}$ curve to the right by $0.5 \tau(0)$, and we see $\varepsilon_{T}(t-$ $0.5 \tau(0)) \simeq \varepsilon(t)$. Or equivalently $\varepsilon(t+\Delta t) \simeq \varepsilon_{T}(t)$ with $\Delta t=0.5 \tau(0)$. This reinforces the point above, that the time taken for the energy to pass through the cascade is one half of the initial large eddy turnover time. 


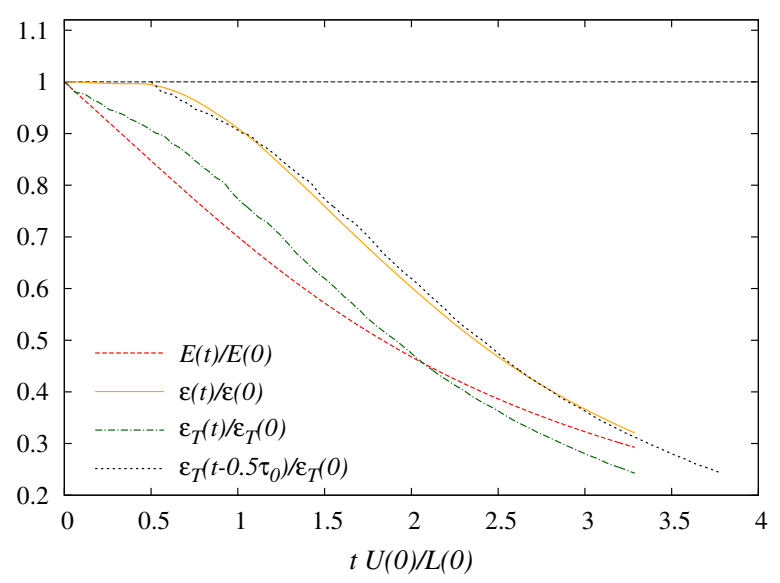

FIG. 7. Time variation of the total energy, dissipation rate and inertial flux for decaying turbulence with an evolved initial condition. The dotted line shows the transfer rate curve shifted to the right by $0.5 \tau(0)$.

\section{A COMPOSITE ONSET CRITERION BASED ON BOTH DISSIPATION AND ENERGY TRANSFER RATES}

As we have seen, the curves for dissipation and energy transfer plotted against time display clear peaks, although in the case of the dissipation rate, there is no peak for $R_{\lambda} \lesssim 25$. This leads us to consider an alternative to using just either the dissipation or the maximum inertial flux. In Figure 8, we plot both the dissipation and the maximum flux against time, for low initial Reynolds numbers. In fact, the peak transfer coincides nicely with an inflection point in $\varepsilon$ for these low Reynolds number runs which do not develop a peak in the dissipation rate. The vertical dotted line on the graph indicates the peak in $\varepsilon_{T}$ for $R_{\lambda}=12.9$. This suggests the idea of a composite evolved time, which we define as:

$$
t_{\varepsilon \mid \Pi}=\left\{\begin{array}{ll}
t_{\varepsilon} & \text { if peak in } \varepsilon \text { exists } \\
t_{\Pi} & \text { otherwise }
\end{array} .\right.
$$

That is, we use the time associated with the peak in the dissipation rate curve, if it exists. Or, failing that, the peak in the curve of the inertial flux.

This provides us with a continuous evolved time. From Figure 9 we see that there is no discontinuity as we go from one regime to the other.

\section{EFFECT OF CHOICE OF EVOLVED TIME CRITERION ON MEASUREMENTS OF THE DIMENSIONLESS DISSIPATION}

As well as considering the traditional method of taking the onset of power-law behaviour as the criterion for evolved time, we consider the effects of the following criteria:

1. $t_{s}$ the time taken for the skewness to reach its peak value.

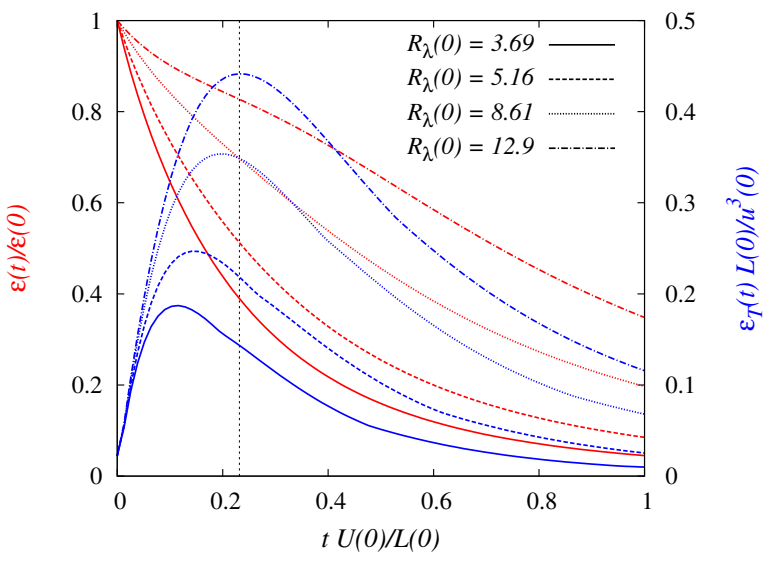

FIG. 8. Comparison of $\varepsilon(t)$ and $\varepsilon_{T}(t)$ for lower Reynolds numbers. Peak in $\varepsilon_{T}$ can be seen to coincide with an inflection in the dissipation rate. The vertical dotted line indicates the position of $t_{\Pi}$ (corresponding to peak $\varepsilon_{T}$ ) for $R_{\lambda}=12.9$.

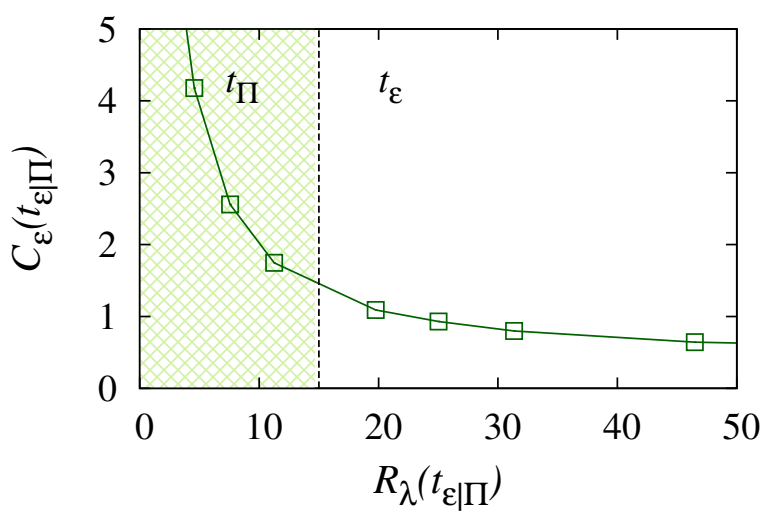

FIG. 9. Continuity of measured dimensionless dissipation rate between two regimes

2. $t_{\Pi}$ the time taken for the inertial transfer rate to reach its peak value.

3. $t_{\varepsilon}$ the time taken for the dissipation rate to reach its peak value.

4. $t_{\varepsilon \mid \Pi}$ a composite time equal to $t_{\varepsilon}$, if peak $\varepsilon$ exists; but equal to $t_{\Pi}$ otherwise.

In Figure 10 we plot the ratio $\varepsilon / \varepsilon_{T}$ against TaylorReynolds number for decaying turbulence, using different criteria for the evolved time. We show results taken at $t_{e}=t_{\varepsilon \mid \Pi}, t_{e}=t_{\Pi}$, and $t_{e}=t_{S}$, along with four arbitrary times at various points during the decay, thus: $t_{e}=5 \tau(0), t_{e}=10 \tau(0), t_{e}=30 \tau(0)$, and $t_{e}=50 \tau(0)$, where $\tau(0)$ is the initial value of the eddy turnover time. As the times $t_{\varepsilon \mid \Pi}$ and $t_{S}$ occur much earlier in the decay than power-law decay of the total energy, it is interesting to to compare how the choice of evolved time affects measurements.

First let us consider what happens with forced turbulence. In this case, energy enters at a rate $\varepsilon_{W}$. At 


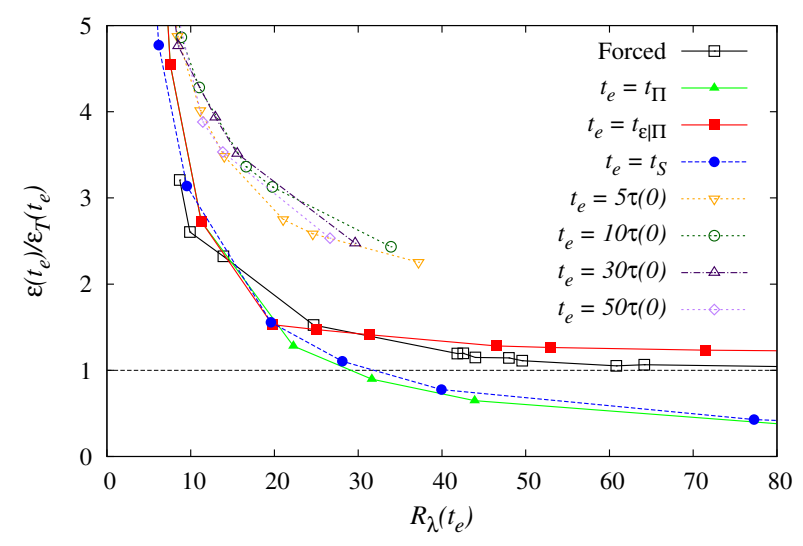

FIG. 10. The ratio $\varepsilon / \varepsilon_{T}$ measured using various criteria for the evolved time, $t_{e}$. Results for forced, stationary turbulence shown for comparison.

the steady state there is a balance of energy in and out: $\varepsilon=\varepsilon_{W}$. At low $R e$, some energy is dissipated by wavenumbers below the inertial range, so we expect to find $\varepsilon_{T}<\varepsilon$. As the Reynolds number is increased, we expect that $\varepsilon_{T} \rightarrow \varepsilon$.

The situation is different for decaying turbulence but also depends on the Reynolds number. Let us begin with the case of high $R e$. We can measure $\varepsilon_{T}(t)>\varepsilon(t)$ in the initial transition period. Due to the finite transit time for energy transfer through the cascade, energy transferred at time $t$ will be dissipated at time $t+\Delta t$. Or $\varepsilon(t+\Delta t)=\varepsilon_{T}(t)$. The turbulence is decaying, so $\varepsilon(t+\Delta t)<\varepsilon(t)$, thus we must have $\varepsilon_{T}(t)<\varepsilon(t)$. Hence the time $t_{\varepsilon}$ is the border between the cases $\varepsilon_{T}(t)>\varepsilon(t)$ and $\varepsilon_{T}(t)<\varepsilon(t)$. One would therefore expect to measure $\varepsilon_{T}\left(t_{\varepsilon}\right)=\varepsilon\left(t_{\varepsilon}\right)$.

Turning to the case of decaying turbulence at low $R e$ : we would still measure $\varepsilon_{T}(t)>\varepsilon(t)$ in the initial transition period. For $t \geqslant t_{\varepsilon}$, we now must have $\varepsilon(t)>\varepsilon_{T}(t)$. The peak dissipation rate is no longer associated with equality of transfer and dissipation, but this is due to finite $R e$.

We may summarise the results of Fig. 10 as follows:

1. Skewness: $\varepsilon_{T}\left(t_{S}\right)>\varepsilon\left(t_{S}\right)$ indicates we are in transition period.

2. Combined: Measure $\varepsilon_{T}\left(t_{\varepsilon \mid \Pi}\right)<\varepsilon\left(t_{\varepsilon \mid \Pi}\right)$ for all Reynolds numbers, but could be asymptoting to unity.

3. Power-law decays: All in agreement with one another; do not asymptote to unity.

4. $\lambda \propto t^{1 / 2}:$ Measurements at $t_{e}=30 \tau(0), 50 \tau(0)$.

We may look more deeply into the measurement of the dimensionless dissipation coefficient by looking at its dependence on time. This illustrates some of the problems involved. We refer to Fig. 11, which shows the time evolution of $C_{\varepsilon}(t)$ for various values of the Taylor-Reynolds number. The measurement time $t_{\varepsilon \mid \Pi}$, as indicated by solid points for the various $R_{\lambda}$,

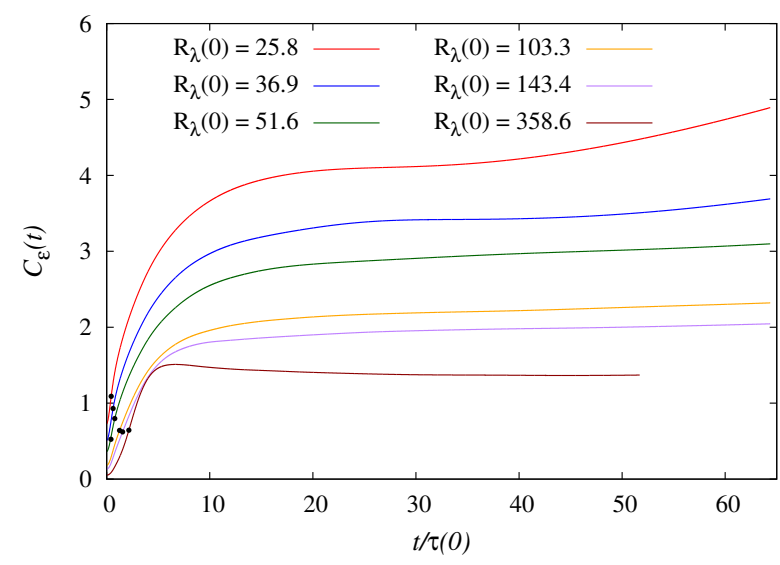

FIG. 11. Time evolution of the dimensionless dissipation rate, $C_{\varepsilon}(t)=\varepsilon(t) L(t) / U^{3}(t)$, plotted against decay time scaled on the initial eddy turnover time $\tau(0)$. Note that the solid black circles are the values of $t_{\varepsilon \mid \Pi}$.

occurs very early in the decay while $C_{\varepsilon}$ is strongly time-dependent.

We note that $C_{\varepsilon}(t)$ develops a plateau from around $t \simeq 10 \tau(0)$. But, while $C_{\varepsilon}(t)$ then remains constant, the Reynolds number still decays. Therefore, the same value of $C_{\varepsilon}(t)$ corresponds to different $R_{\lambda}(t)$ and a plot of $C_{\varepsilon}(t)$ against $R_{\lambda}(t)$ for different $t$ will have their curves shifted.

The behaviour $C_{\varepsilon}(t) \sim$ constant observed in Fig. 11 for a period of the decay requires:

$$
\frac{L}{\lambda} \sim R_{\lambda}, \quad\left(\frac{L}{\lambda}\right)^{2} \sim R_{L}, \quad \text { or } \quad R_{L} \sim R_{\lambda}^{2} .
$$

Fig. 12 shows $L / \lambda$ plotted against $R_{\lambda}$ during the decay. We may observe a region at lower Reynolds number $R_{\lambda}$ where the relationship is approximately linear (as indicated by the dashed line). This behaviour was found experimentally for regular grids by Valente and Vassilicos [31] for low $R_{\lambda}$ far downstream, and followed a region where $L / \lambda \sim$ constant, in which case $C_{\varepsilon} \sim 1 / R_{L} \sim 1 / R_{\lambda}$. Their results for a fractal grid show good agreement, but far fewer points were plotted (see the open squares in their figure 5).

Lastly, we consider the question of how the usual curves of $C_{\varepsilon}$ against $R_{\lambda}$ are affected by our choice of onset criterion. This is shown in Fig. 13, which makes a comparison of $C_{\varepsilon}\left(t_{e}\right)$ against $R_{\lambda}\left(t_{e}\right)$ for different evolved time criteria. When we use the peak skewness, $t_{S}$ or the peak inertial transfer time $t_{\Pi}$, the dimensionless dissipation rate appears to approach zero as $R_{\lambda}$ increases. However, if we take our preferred criterion, based on the dissipation, $t_{\varepsilon \mid \Pi}$ then the dimensionless dissipation rate appears to match the forced case (which is plotted for comparison).

If we go to later measurement times, then we find very different behaviour. For choices of $t_{e}$ in the range $3 \tau(0) \leqslant t_{e} \leqslant 50 \tau(0)$, we find that the curves cluster together and follow a similar profile to the forced case, only translated up the $C_{\varepsilon}$-axis. 


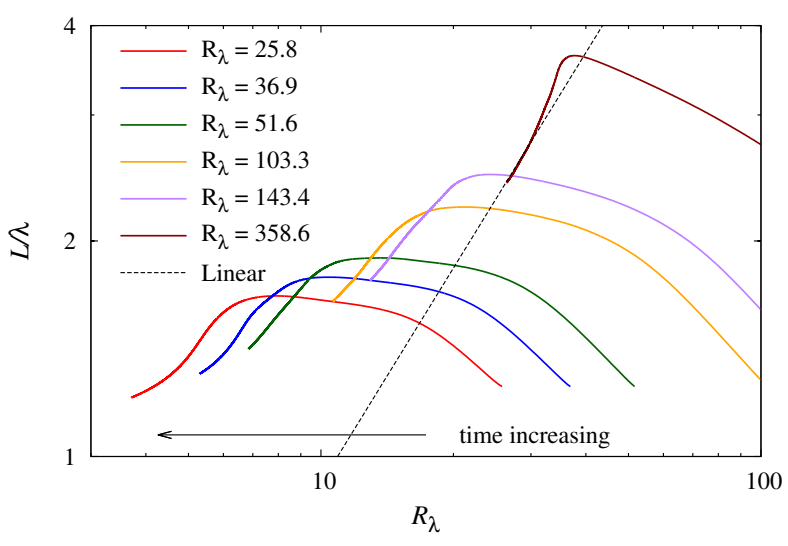

FIG. 12. Variation of the ratio of integral scale $L$ to microscale $\lambda$ during the decay, as a function of TaylorReynolds number. Since $R_{\lambda}$ falls off during the decay, time runs from right to left. Linear behaviour for each curve is shown by a region parallel to the dotted line.

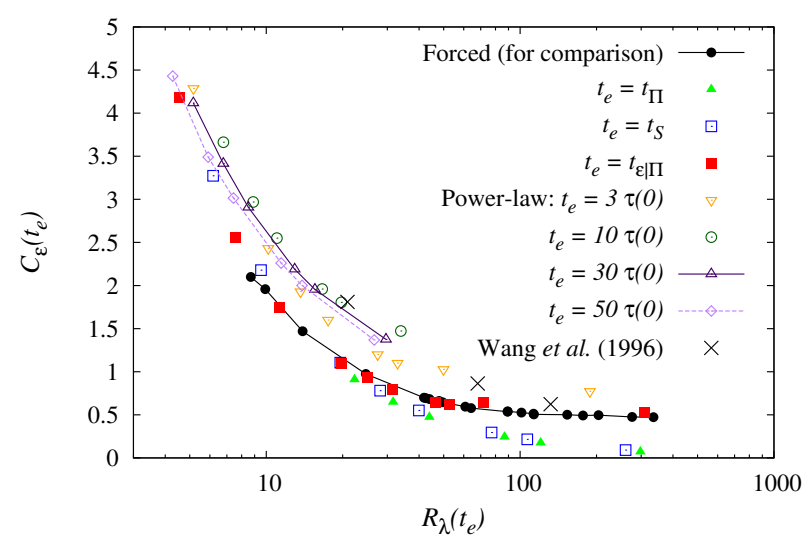

FIG. 13. The Reynolds number variation of the dimensionless dissipation rate for decaying turbulence. Plotted for various choices of the evolved time $t_{e}$, along with results for the forced case and decay data from Wang et al [16], for comparison.

In all, we conclude that the asymptote for decaying turbulence is in the range $0 \leqslant C_{\varepsilon, \infty}\left(t_{e}\right) \lesssim 1.2$, depending on the choice of evolved time.

\section{CONCLUSION}

We have been mainly concerned with the way in which choice of an evolution time $t_{e}$ affects the shape of the curve of $C_{\varepsilon}$ when plotted against $R_{\lambda}$. Figure 13 is the key result here. It is clear that different choices of evolution time can produce radically different results. A failure to recognise this presents a problem for the comparison of one investigation with another. Indeed, this degree of variation is quite sufficient to explain the spread of results commonly found in surveys.
We would suggest that meaningful comparisons would need one to compare the detailed evolution (with time or space) of the dissipation rate (see Figure 3) or the dimensionless dissipation rate (see Figure 11). It would be more satisfactory if a consensus could arise regarding a choice of onset criterion, although we cannot dismiss the possibility that separate criteria might be needed for different statistical quantities.

Our preferred criterion $t_{\varepsilon \mid \Pi}$ has some physical appeal in that it is determined from primary statistical quantities rather than the secondary characteristic of an apparent power law decay. Moreover, although it is a composite form, it displays continuous behaviour as a criterion in a plot of dimensionless dissipation versus Taylor-Reynolds number as seen in Fig. 9. Thus it links the low-Reynolds number flows with the highReynolds number flows, as defined in Fig. 3. It should perhaps be emphasised that these low-Reynolds number flows cannot be dismissed as being nonturbulent, as we have previously reported for low Reynolds numbers in forced turbulence [49]. From the results shown in Figures 4 and 5, it can be seen that even at these low Reynolds numbers, there is a development away from the initial Gaussian state, with the growth of inertial transfer and skewness from zero, to reach a peak value.

However, if we are to make meaningful comparisons between decaying and stationary turbulence, then a more fundamental justification is needed. As mentioned in Section 1, recently McComb and Fairhurst [5] showed by means of asymptotic expansion of structure functions in powers of the inverse Reynolds number, that the time derivative in the Karman-Howarth equation has an irreducible remainder which does not vanish in the limit of large Reynolds numbers or at particular length scales. Accordingly, they concluded that Kolmogorov's hypothesis of local stationarity cannot be correct. The question then arises: how large is the error if we neglect the term? In principle, this can be answered by a direct comparison of $C_{\varepsilon, \infty}$ in both forced and decaying flows. But for this comparison to be meaningful we need an agreed definition (and hence value) of $t_{e}$.

If we insist on our composite criterion for the purposes of studying the dimensionless dissipation, then we have to accept that there is a second transition (at a later time) to power-law behaviour. This in itself should not be too surprising. For instance, some simulations of forced turbulence have shown a transition to turbulence from a Beltrami flow at a specific Reynolds number, followed by a second transition to scaling behaviour at a higher Reynolds number [49]. Of course our presentation here is purely in terms of physical reasoning. Ideally the problem could be solved mathematically as an example of a phase transition. However, even although the study of hydrodynamic stability has been going on for 140 years, and is now an immense field of activity (see the superb review by Zhou [50]), we are not aware of any possibility of a mathematical theory of the development of isotropic turbulence in free decay.

We conclude by restating our general position. We 
are studying the decay of the (initially) random motion of an incompressible, viscous fluid. We extend our analysis by using DNS, and our method of doing this is part of a long established general paradigm. Thus, when we explore the possibility of an alternative starting point, it is more from the point of view of statistical physics than from any conviction that there might be something unsatisfactory about our initial spectra. Because in this paradigm, the qualitative (and quantitative) performance of the simulations, in terms of statistical parameters, spectra, and fluxes, is very well established. Morever, the other investigations that are summarised in our Figure 1 are also part of the same paradigm. Accordingly, all our results are applicable to these investigations, for we are comparing like with like.

\section{ACKNOWLEDGEMENT}

The authors thank Professor Arjun Berera for many stimulating discussions of this work. SRY is grateful for the support of an STFC studentship while this work was undertaken, and is currently supported by the UK EPSRC grant EP/N028694. Simulations were performed on the Eddie HPC cluster hosted by the Edinburgh Parallel Computing Centre (EPCC). Data are available online [http://dx.doi.org/10.15129/64a4a0427d0d-48ce-8afa-21f9883d1e84].

\section{Appendix A: Results for power-law behaviour from measurements of the local slope}

This is a confused and rather controversial topic. For recent reviews of the subject, we suggest the books [26] and [37]. Here, we give a brief introduction to the topic and present a summary of representative values of decay exponents in Table III. In this way, it can be seen how our own results fit in with the rest of the field.

The topic is still dominated by the classic theories of Kolmogorov [51], with $n=10 / 7 \sim 1.43$ and Saffman [52], with $n=6 / 5 \sim 1.2$. These are rival theories, either of which may apply, depending on the initial conditions determining whether the Loitiansky integral or the Saffman-Birkhoff integral is an invariant. The former case is sometimes referred to as Batchelor turbulence and the latter as Saffman turbulence.[53] This situation can be understood in terms of the infrared behaviour of the energy spectrum $E(k, t)$. As is well known, this can be written as a Taylor polynomial at small wavenumbers, thus:

$$
E(k, t)=E_{2}(t) k^{2}+E_{4}(t) k^{4}+\ldots,
$$

where $E_{2}(t)$ is the Saffman-Birkhoff integral and $E_{4}(t)$ is the Loitsiansky integral. In fact a recent study of this problem has shown that $E_{2}(t)=0$ is an exact result [54], and hence the Saffman-Birkhoff integral is zero.

The most significant (relatively) modern experimental studies of this topic were probably those due to Comte-Bellot and Corrsin in 1966 and 1971 [55] and [56]. These established values of the decay exponent in the range $1.16 \leqslant n \leqslant 1.37$. This was followed by Mohamed and LaRue [57] and later by Skrbek and Stalp [58]. The first of these investigated the effect of initial conditions, and concluded that they only affected the decay coefficient and not the exponent or the virtual origin. A particularly interesting feature of the work by Skrbek and Stalp was their investigation of the relationship between the finite size of the test-section and the largest eddies. They discussed a saturation effect in which the nature of the decay changed after the largest eddies had grown to the same size as the 'box'. This is now being investigated in numerical simulations too, where finite size and finite Reynolds number effects are being studied: see the recent work by Thornber [59], and Meldi and Sagaut [60].

We may also mention the study of the effects of increasing the wavenumber corresponding to the peak value of the initial spectrum by Ishida, Davidson and Kaneda [61]. This investigation uses an initial spectrum similar to our equation (10). Custom and practice normally dictate that this wavenumber (denoted by $k_{p}$ in Ishida et al.) is taken to be as small as possible in order to allow turbulence to develop which is characteristic of the Navier-Stokes equations, rather than of the initial conditions. It is not simply a increase in the resolution of the integral lengthscale. However, Ishida et al. consider values of $k_{p}$ up to 80, as compared with our equivalent of $k_{p}=5$, in order to study the low- $k$ behaviour of the spectrum. Here we 

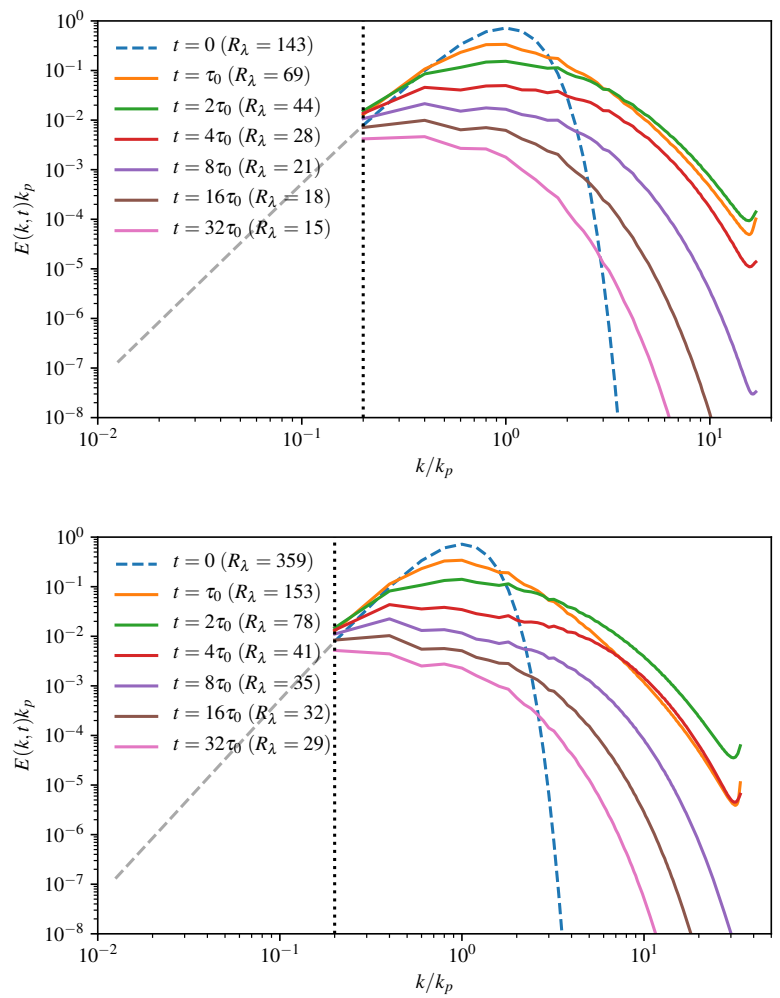

FIG. 14. Time evolution of the energy spectrum for one of our $256^{3}$ simulations (top) and our $512^{3}$ simulation (bottom), plotted for comparison to Figure 4 from Ishida et al. [61].

replot our results for the time evolution of the energy spectrum as Figure 14 in order to make a comparison with Figures 4, 7 and 9 from Ishida et al. It may be seen that the behaviour is quite similar. In addition, our results for the energy decay exponent in Figure 18 may be seen to be in agreement with those of Ishida et $a l$. as given in their Figures 3 and 6 . However, Ishida et al. do not present any results for transfer spectra or skewness factor to indicate that their turbulence is well-developed, nor any data for the dissipation rate, velocity, or lengthscales in order to facilitate a comparison of values of $C_{\varepsilon}$.

More recent experimental studies include those of Vassilicos and co-workers [28]-[31] and by Krogstad and Davidson [34]. The latter measured decay exponents in a particularly large wind tunnel and concluded that, when they made allowance for a weak decay of the dimensionless dissipation rate, the turbulence was of the Saffman type. They also investigated turbulence generated by multiscale grids [32], and concluded that the resulting decay was almost identical to that behind conventional grids. This was in contrast to what had been found by Vassilicos and co-workers. A subsequent paper by Valente and Vassilicos [30] reaffirmed the untypical properties of turbulence generated by multiscale grids. Decay exponents for all three investigations are listed in Table III.

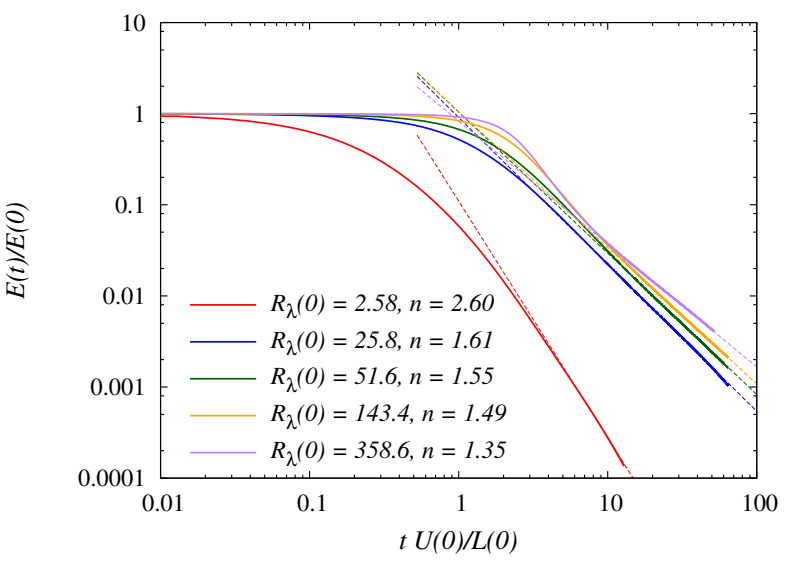

FIG. 15. Identification of power-law decay of the total energy. The total energy is plotted against time on logscales, such that a period satisfying power-law decay can be identified by a straight line.

\section{Onset criteria based on power-law decay}

We begin with the direct determination of powerlaw decay and then move on to the indirect method based on the Taylor microscale. The turbulent kinetic energy and dissipation rate decay are generally found to decay with time as

$$
E(t) \propto t^{-n}, \quad \varepsilon(t) \propto t^{-n-1} .
$$

In Figure 15, we show the decay curves for the energy $E(t)$ divided by its initial value, for a range of initial values of the Reynolds number, ranging over $2.58 \leqslant R_{\lambda} \leqslant 358.6$. The figure also shows the measured exponents for the decay and these were found to decrease with increasing Reynolds number. Table III summarises the experimental and theoretical situation regarding decay exponents. It can be seen from there that our values for exponents are not out of line with the field in general. In view of the lack of consensus in this field, we can make no stronger statement than that.

In Fig. 16 we illustrate the way in which we determined the decay exponent by measuring the local slope of the decay curves. This is based on the procedure

$$
-n(t)=\frac{d \log E}{d \log t}=\frac{t}{E} \frac{d E}{d t} .
$$

Evidently when we plot $n(t)$ against time on linear scales, as in Fig. 16, a plateau corresponds to a region of power-law decay.

\section{The onset of $t^{1 / 2}$ scaling of $\lambda$}

As an alternative criterion, we can make use of this well known exact result for the decay of the Taylor microscale $\lambda$ in isotropic decaying turbulence, as referred to in Section 3.1. 


\begin{tabular}{lll} 
Reference & $n$ & Comments \\
\hline \hline Kolmogorov [51] & $n=10 / 7$ & Notes that [62] found $E \propto t^{-n}$ but did not find $n$ \\
Batchelor [63] & $n=5 / 2$ & In the final period of the decay \\
Saffman [52] & $n=6 / 5$ & \\
\hline Mohamed and & $n=1$ and $1.16 \leqslant n \leqslant 1.37$ & Review of experimental results \\
LaRue [57] & $n=1.42,1.33,1.10,0.95$ & Virtual origin $x_{0} / M_{u}=0,2,4,6$ \\
& $n=1.24 \leqslant n \leqslant 1.33$ & Optimised origin $x_{0}-$ table 4. \\
\hline Yu et al [64] & $n=1.38-1.85$ & LB: $R_{\lambda}=2.3-22.5\left(E(k) \sim k^{4}\right)$ \\
{$[65]$} & $n=1.1-1.52$ & DNS: $R_{\lambda}=0-30\left(E(k) \sim k^{2}\right)$ \\
[66] & $n=1.0-3.0$ & DNS: $R_{\lambda}=10-50\left(E(k) \sim k^{2}\right)$ \\
{$[57]$} & $n=1.285-1.309$ & Exp: $R_{\lambda}=28.4-43.9\left(E(k) \sim k^{2}\right)$ \\
[67] & $n=1.3-1.8$ & Exp: $R_{\lambda}=4.4-5.4\left(E(k) \sim k^{2}\right)$ \\
\hline Krogstad and & $1.15<n<1.29$ & Comte-Bellot and Corrsin $(1966)$ \\
Davidson [34] & $n \sim 1.34$ & Warhaft and Lumley $(1978)$ \\
& $1.09<n<1.19$ & Find an average of $n=1.13 \pm 0.02$ \\
\hline Valente and & $n=1.25-1.36$ & Regular grid \\
Vassilicos [30] & $n=1.93-2.57$ & Fractal grid \\
\hline Krogstad and & $n=1.13 \pm 0.02$ & Conventional grid (regression) \\
Davidson [32] & $n=1.14 \pm 0.02$ & Conventional grid (local slope) \\
& $n=1.17 \pm 0.04$ & Conventional grid (maximum decay) \\
& $n=1.12 \pm 0.02$ & Fractal grid 1 (regression) \\
& $n=1.17 \pm 0.02$ & Fractal grid 1 (local slope) \\
& $n=1.19 \pm 0.03$ & Fractal grid 1 (maximum decay) \\
& $n=1.25 \pm 0.02$ & Fractal grid 2 (regression) \\
& $n=1.25 \pm 0.02$ & Fractal grid 2 (local slope) \\
& $n=1.23 \pm 0.03$ & Fractal grid 2 (maximum decay) \\
& $n=1.49$ & $R_{\lambda}(0)=143.4$ \\
Our DNS data & $n=1.35$ & $R_{\lambda}(0)=358.6$ \\
& &
\end{tabular}

TABLE III. A summary of values, both theoretical and experimental, of the decay exponent $n$ such that $U^{2} \propto t^{-n}$, from the literature. Note that 'LB' stands for Lattice Boltzmann, 'DNS' stands for Direct Numerical Simulation, and 'Exp' stands for experimental.

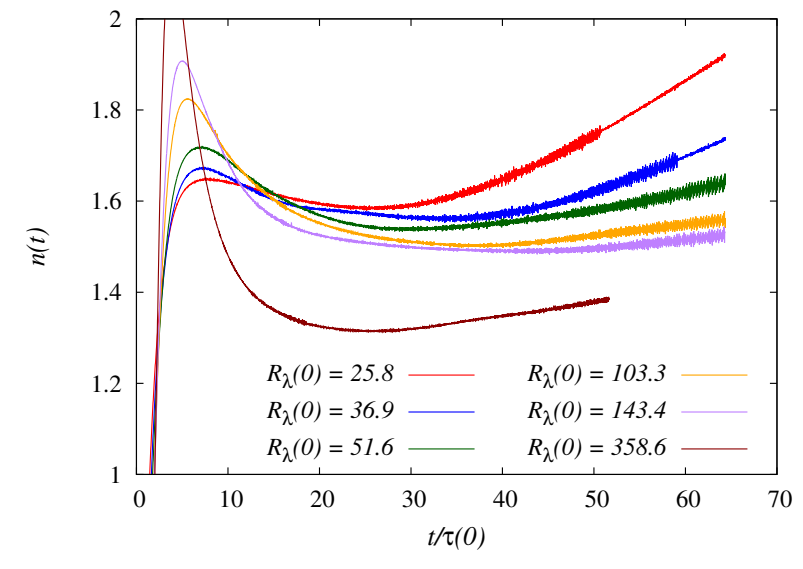

FIG. 16. The local slope for the decay of the total energy, plotted against (scaled) time. A plateau indicates a region of power-law decay.

Using standard relationships for decay and dissipation rates in isotropic turbulence (e.g. see [37]) we may write:

$$
\frac{d E}{d t}=\frac{3}{2} \frac{d U^{2}}{d t}=-\varepsilon=-\frac{15 \nu_{0} U^{2}}{\lambda^{2}}
$$

Then, for power-law decay, where $U^{2} \propto t^{-n}$, we have

$$
\begin{aligned}
-n t^{-n-1} & =-\frac{10 \nu_{0}}{\lambda^{2}} t^{-n} \\
\lambda^{2} & =\frac{10 \nu_{0}}{n} t \quad \Longrightarrow \quad \lambda \propto \sqrt{t},
\end{aligned}
$$

for all exponents $n$. We note that Figure 17 shows $\sqrt{t}$-scaling for $t \gtrsim 25 \tau(0)$, where $\tau(0)=L(0) / U(0)$. Clearly this rules out exponential decay because, if $U^{2} \propto \exp (-\alpha t)$, then we have the well known result

$$
\begin{aligned}
-\alpha \exp (-\alpha t) & =-\frac{10 \nu_{0}}{\lambda^{2}} \exp (-\alpha t) \\
\lambda^{2} & =\frac{10 \nu_{0}}{\alpha} \quad \Longrightarrow \quad \lambda=\text { constant. }
\end{aligned}
$$

The idea of using the behaviour of the Taylor microscale as a way of obtaining the decay exponent for the energy has been explored by various workers in the field. For example, Huang and Leonard [66], Burattini et al [68], and Lavoie et al [69]. In the interests of completeness, we present some results of our own for this procedure in Appendix B. However, we note here that the investigation of Burattini et al [68] is of particular interest to us as they found that their results for $C_{\varepsilon}$ in free decay were comparable to those for forced turbulence. 


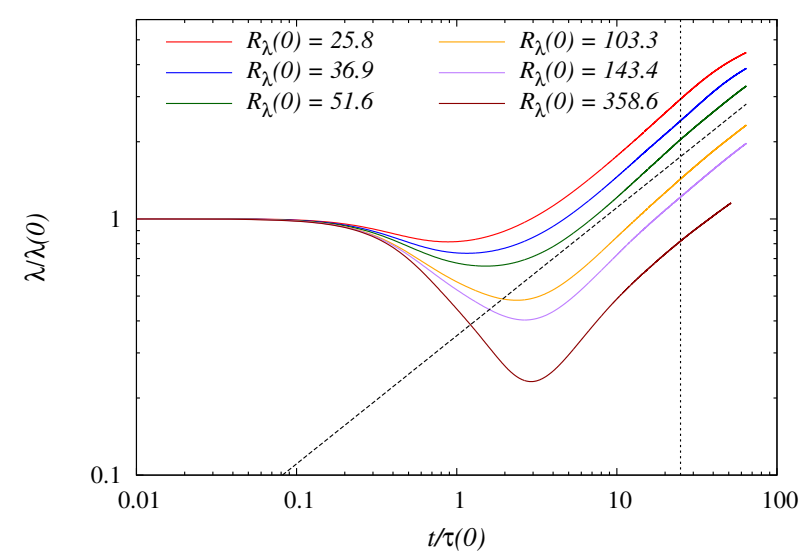

FIG. 17. Identification of $t^{1 / 2}$ behaviour of the Taylor microscale, $\lambda$, for several different Reynolds numbers. The vertical dotted line indicates the time after which we appear to observe $\sqrt{t}$ scaling, as shown by the dashed line.

\section{Appendix B: Energy decay exponent from the decay of the Taylor microscale}

As mentioned in Appendix A, we can obtain a value of the exponent $n$ from the decay of the Taylor microscale. We do this by rearranging the relationship for $\lambda^{2}(t)$, as given in equation (A4), and by introducing a virtual origin $t_{0}$. In practice, uncertainty about the value of the virtual origin requires iterative methods. We will retain it in our formulation but for sake of simplicity we will set it equal to zero in calculations.

We find that

$$
n(t)=\frac{10 \nu_{0}}{\lambda^{2}(t)}\left(t-t_{0}\right)
$$

where $t_{0}$ is the virtual origin of the power-law decay. Figure 18 shows $n(t)$ calculated in this manner with $t_{0}=0$. The dashed horizontal line indicates the Kolmogorov value of $10 / 7$. This is a simplified version of what is considered later, in Figure 20.

In order to find the decay exponent $m$ of the microscale, we proceed much as we did for the decay of the energy. Consider $\lambda(t) \sim t^{m}$, where $m=1 / 2$ corresponds to power-law decay of the total energy. As before, we measure the local slope,

$$
m(t)=\frac{d \log \lambda}{d \log t}=\frac{t}{\lambda} \frac{d \lambda}{d t} .
$$

In Figure 19, we plot the resulting $m(t)$ against time. A plateau at $1 / 2$, shown by the horizontal dashed line on the graph, would indicate a region of power-law decay of the total energy.

We can now obtain the energy exponent $n$ from the behaviour of $\lambda$. To do this, we take a derivative with respect to $t$ of equation (A4), to obtain:

$$
\frac{d \lambda^{2}(t)}{d t}=\frac{10 \nu_{0}}{n}
$$

from which we deduce that

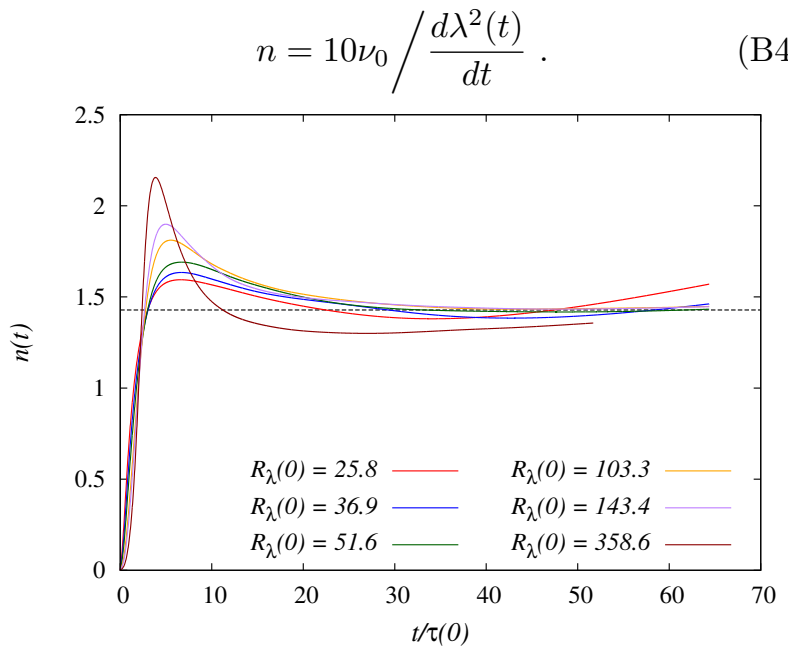

FIG. 18. Evaluation of the energy decay exponent using the Taylor microscale, $n=10 \nu\left(t-t_{0}\right) / \lambda^{2}(t)$ with $t_{0}=0$. The horizontal line indicates the Kolmogorov value of $n=$ $10 / 7$.

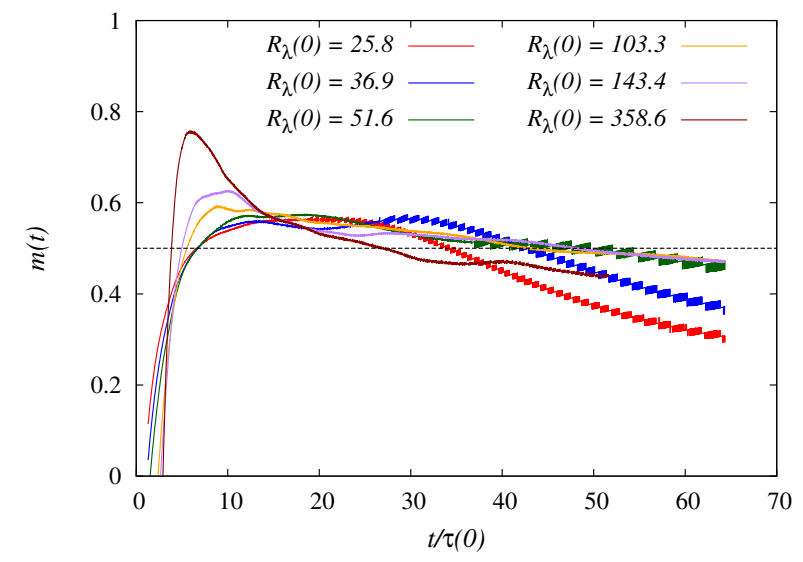

FIG. 19. The local slope for the time-variation of the Taylor microscale, $\lambda$, such that $\lambda(t) \propto t^{m}$. A plateau at 0.5 , as indicated by the horizontal dashed line, would represent a region of power-law decay for the total energy.

Note that this form is not dependent on the time when power-law decay starts, $t_{0}$. This is plotted in the lefthand figure of Figure 20.

Alternatively, we could take $U^{2} \sim\left(t-t_{0}\right)^{-n(t)}$, in which case for equation (A4) we have

$$
\frac{\partial}{\partial t}\left(n(t) \log \left(t-t_{0}\right)\right)=\frac{10 \nu_{0}}{\lambda^{2}(t)},
$$

which we integrate (with $n(0)=0$ ) to find

$$
n(t)=\frac{10 \nu_{0}}{\log \left(t-t_{0}\right)} \int_{0}^{t} \frac{d s}{\lambda^{2}(s)} .
$$

This is clearly dependent on $t_{0}$. Note: Fukayama et al [38] comment on numerical integration being more stable than numerical differentiation. The results of this procedure are presented in the right-hand figure of Figure 20, with $t_{0}=0$. Increasing $t_{0}$ would have the effect of lowering the curves. 

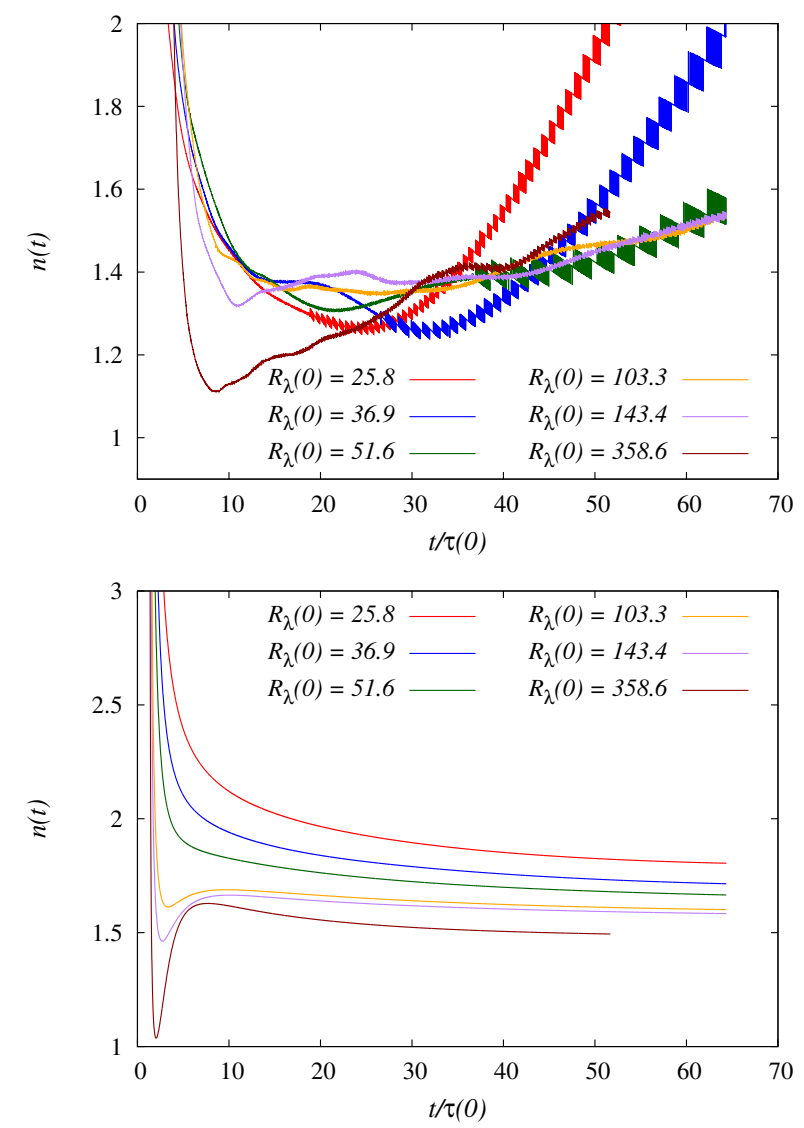

FIG. 20. Calculation of the power-law decay exponent for the total energy. (Left) Calculated from $d \lambda^{2} / d t$. (Right) Calculated using $(1 / \log t) \int_{0}^{t} d s 1 / \lambda^{2}$.

[1] W. David McComb, Arjun Berera, Matthew Salewski, and Sam R. Yoffe. Taylor's (1935) dissipation surrogate reinterpreted. Phys. Fluids, 22:61704, 2010.

[2] Thomas S. Lundgren. Kolmogorov two-thirds law by matched asymptotic expansion. Phys. Fluids, 14:638, 2002.

[3] W. D. McComb, A. Berera, S. R. Yoffe, and M. F. Linkmann. Energy transfer and dissipation in forced isotropic turbulence. Phys. Rev. E, 91:043013, 2015.

[4] M. F. Linkmann, A. Berera, W. D. McComb, and M. E. McKay. Nonuniversality and Finite Dissipation in Decaying Magnetohydrodynamic Turbulence. Phys. Rev. Lett., 114:235001, 2015.

[5] W. D. McComb and R. B. Fairhurst. The dimensionless dissipation rate and the Kolmogorov (1941) hypothesis of local stationarity in freely decaying isotropic turbulence. J. Math. Phys., 59:073103, 2018.

[6] A. N. Kolmogorov. The local structure of turbulence in incompressible viscous fluid for very large Reynolds numbers. C. R. Acad. Sci. URSS, 30:301, 1941.

[7] A. N. Kolmogorov. Dissipation of energy in locally isotropic turbulence. C. R. Acad. Sci. URSS, 32:16, 1941.

[8] G. K. Batchelor. The theory of homogeneous turbulence. Cambridge University Press, Cambridge, 1st edition, 1953.
[9] G. I. Taylor. Statistical theory of turbulence. Proc. R. Soc., London, Ser. A, 151:421, 1935.

[10] G. K. Batchelor. The theory of homogeneous turbulence. Cambridge University Press, Cambridge, 2nd edition, 1971.

[11] K. R. Sreenivasan. On the scaling of the turbulence dissipation rate. Phys. Fluids, 27:1048, 1984.

[12] K. R. Sreenivasan. An update on the energy dissipation rate in isotropic turbulence. Phys. Fluids, 10:528, 1998.

[13] J. Jiménez, A. A. Wray, P. G. Saffman, and R. S. Rogallo. The structure of intense vorticity in isotropic turbulence. J. Fluid Mech., 255:65, 1993.

[14] P. K. Yeung and Y. Zhou. Universality of the Kolmogorov constant in numerical simulations of turbulence. Phys. Rev. E, 56:1746, 1997.

[15] N. Cao, S. Chen, and G. D. Doolen. Statistics and structures of pressure in isotropic turbulence. Phys. Fluids, 11:2235-2250, 1999.

[16] L.-P. Wang, S. Chen, J. G. Brasseur, and J. C. Wyngaard. Examination of hypotheses in the Kolmogorov refined turbulence theory through highresolution simulations. Part 1. Velocity field. J. Fluid Mech., 309:113, 1996.

[17] P. Burattini, P. Lavoie, and R. Antonia. On the normalised turbulence energy dissipation rate. Phys. Flu- 
ids, 17:98103, 2005

[18] T. Gotoh, D. Fukayama, and T. Nakano. Velocity field statistics in homogeneous steady turbulence obtained using a high-resolution direct numerical simulation. Phys. Fluids, 14:1065, 2002.

[19] Y. Kaneda, T. Ishihara, M. Yokokawa, K. Itakura, and A. Uno. Energy dissipation and energy spectrum in high resolution direct numerical simulations of turbulence in a periodic box. Phys. Fluids, 15:L21, 2003.

[20] D. A. Donzis, K. R. Sreenivasan, and P. K. Yeung. Scalar dissipation rate and dissipative anomaly in isotropic turbulence. J. Fluid Mech., 532:199-216, 2005.

[21] B. R. Pearson, P. A. Krogstad, and W. van de Water. Measurements of the turbulent energy dissipation rate. Phys. Fluids, 14:1288, 2002.

[22] B. R. Pearson, T. A. Yousef, N. E. L. Haugen A. Brandenburg, and P. A. Krogstad. Delayed correlation between turbulent energy injection and dissipation. Phys. Rev. E, 70:56301, 2004.

[23] W. J. T. Bos, L. Shao, and J.-P. Bertoglio. Spectral imbalance and the normalized dissipation rate of turbulence. Phys. Fluids, 19:45101, 2007.

[24] L. Mydlarski and Z. Warhaft. On the onset of highReynolds-number grid-generated wind tunnel turbulence. J. Fluid Mech., 320:331-368, 1996.

[25] H. Tennekes and J. L. Lumley. A first course in turbulence. MIT Press, Cambridge, Mass., 1972.

[26] P. A. Davidson. Turbulence. Oxford University Press, 2004.

[27] P. Sagaut and C. Cambon. Homogeneous Turbulence Dynamics. Cambridge University Press, Cambridge, 2008.

[28] R. E. Seoud and J. C. Vassilicos. Dissipation and decay of fractal-generated turbulence. Phys. Fluids, 19:105108, 2007

[29] N. Mazellier and J. C. Vassilicos. The turbulence dissipation constant is not universal because of its universal dependence on large-scale flow topology. Phys. Fluids, 20:15101, 2008.

[30] P. C. Valente and J. C. Vassilicos. The decay of turbulence generated by a class of multiscale grids. $J$. Fluid Mech., 687:300-340, 2011.

[31] P. C. Valente and J. C. Vassilicos. Universal Dissipation Scaling for Nonequilibrium Turbulence. Phys. Rev. Lett., 108:214503, 2012.

[32] P. A. Krogstad and P. A. Davidson. Freely decaying, homogeneous turbulence generated by multi-scale grids. J. Fluid Mech., 680:417-434, 2011.

[33] L. Djenidi, N. Lefeuvre, M. Kamruzzaman, and R. A. Antonia. On the normalized dissipation parameter $C_{\epsilon}$ in decaying turbulence. J. Fluid Mech., 817:63, 2017.

[34] P. A. Krogstad and P. A. Davidson. Is grid turbulence Saffman turbulence? J. Fluid Mech., 642:373-394, 2010.

[35] W. D. McComb, A. Hunter, and C. Johnston. Conditional mode-elimination and the subgrid-modelling problem for isotropic turbulence. Phys. Fluids, 13:2030, 2001.

[36] M. Meldi and P. Sagaut. Investigation of anomalous very fast decay regimes in homogeneous isotropic turbulence. J. of Turbulence, 390-413:19, 2018.

[37] W. David McComb. Homogeneous, Isotropic Turbulence: Phenomenology, Renormalization and Statistical Closures. Oxford University Press, 2014.

[38] D. Fukayama, T. Oyamada, T. Nakano, T. Gotoh, and K. Yamamoto. Longitudinal structure functions in decaying and forced turbulence. J. Phys. Soc.
Japan, 69:701, 2000.

[39] For quantitative consideration of the resolution of the dissipation length scales, see Fig. 2 in [35] and Fig. 5.2 of [44].

[40] G. I. Taylor and A. Green. Mechanism of the Production of Small Eddies from Large Ones. Proc. Roy. Soc. London A, 158(895):499-521, 1937.

[41] M. E. Brachet, D. I. Meiron, S. A. Orszag, B. G. Nickel, R. H. Morf, and U. Frisch. Small-scale structure of the Taylor-Green vortex. J. Fluid Mech., 130:411-452, 1983.

[42] hit3d is available from http://code.google.com/p/hit3d/.

[43] W. D. McComb, S. R. Yoffe, M. F. Linkmann, and A. Berera. Spectral analysis of structure functions and their scaling exponents in forced isotropic turbulence. Phys. Rev. E, 90:053010, 2014.

[44] S. R. Yoffe. Investigation of the transfer and dissipation of energy in isotropic turbulence. PhD thesis, University of Edinburgh, 2012.

[45] T. Ishihara, T. Gotoh, and Y. Kaneda. Study of highReynolds number isotropic turbulence by direct numerical simulation. Ann. Rev. Fluid Mech., 41:165, 2009.

[46] L. Machiels. Predictability of small-scale motion in isotropic fluid turbulence. Phys. Rev. Lett., 79(18):3411-3414, 1997.

[47] A. Vincent and M. Meneguzzi. The spatial structure and statistical properties of homogeneous turbulence. J. Fluid Mech., 225:1-20, 1991.

[48] R. M. Kerr. Higher-order derivative correlations and the alignment of small-scale structures in isotropic numerical turbulence. J. Fluid Mech., 153:31-58, 1985.

[49] W. D. McComb, M. F. Linkmann, A. Berera, S. R. Yoffe, and B. Jankauskas. Self-organization and transition to turbulence in isotropic fluid motion driven by negative damping at low wavenumbers. J. Phys. A Math.Theor., 48:25FT01, 2015.

[50] Y. Zhou. Rayleigh-Taylor and Richmyer-Meshkov instability induced flow, turbulence and mixing. I. Physics Reports, 720-722:1-136, 2017.

[51] A. N. Kolmogorov. On the degeneration of isotropic turbulence in an incompressible fluid. C. R. Acad. Sci. URSS, 31:538, 1941.

[52] P. G. Saffman. The large-scale structure of homogeneous turbulence. J. Fluid Mech, 27:581, 1967.

[53] Indeed Batchelor [63] also predicted $n=5 / 2$, but that was for the final period of the decay, when viscous forces become dominant.

[54] W. D. McComb. Infrared properties of the energy spectrum in freely decaying isotropic turbulence. Phys. Rev. E, 93:013103, 2016.

[55] G. Comte-Bellot and S. Corrsin. The use of a contraction to improve the isotropy of grid-generated turbulence. J. Fluid Mech., 25:657, 1966.

[56] G. Comte-Bellot and S. Corrsin. Simple Eulerian time correlation of full- and narrow-band velocity signals in grid-generated, 'isotropic' turbulence. J. Fluid Mech., 48:273-337, 1971.

[57] M. S. Mohamed and J. C. LaRue. The decay power law in grid-generated turbulence. J. Fluid Mech., 219:195-214, 1990.

[58] L. Skrbek and S. R. Stalp. On the decay of homogeneous isotropic turbulence. Phys. Fluids, 12:1997, 2000.

[59] B. Thornber. Impact of domain size and statistical errors in simulations of homogeneous decaying turbulence and the Richmyer-Meshkov instability. Phys. 
Fluids, 28:045106, 2016.

[60] M. Meldi and P. Sagaut. Turbulence in a box: Quantification of large-scale resolution effects in isotropic turbulence free decay. J. Fluid Mech., 818:697-715, 2017.

[61] T. Ishida, P. A. Davidson, and Y. Kaneda. On the decay of isotropic turbulence. J. Fluid Mech., 564:455$475,2006$.

[62] T. von Karman and L. Howarth. On the statistical theory of isotropic turbulence. Proc. Roy. Soc. Lond. A, 164:192, 1938.

[63] G K Batchelor. Energy Decay and Self-Preserving Correlation Functions in Isotropic Turbulence. $Q$. Appl. Math., 6:97-116, 1948.

[64] H. Yu, S. S. Girimaji, and L.-S. Luo. Lattice Boltzmann simulations of decaying homogeneous isotropic turbulence. Phys. Rev. E, 71:016708, 2005.
[65] N. N. Mansour and A. A. Wray. Decay of isotropic turbulence at low Reynolds number. J. Fluid Mech., 6:808-814, 1994.

[66] M.-J. Huang and A. Leonard. Power-law decay of homogeneous turbulence at low Reynolds numbers. Phys. Fluids, 6(11):3765, 1994.

[67] M. R. Smith, R. J. Donnelly, N. Goldenfeld, and W. F. Vinen. Decay of Vorticity in Homogeneous Turbulence. Phys. Rev. E, 71:2583, 1993.

[68] P. Burattini, P. Lavoie, A. Agrawal, L. Djenidi, and R. Antonia. Power law of decaying homogeneous isotropic turbulence at low Reynolds number. Phys. Rev. E, 73:066304, 2006.

[69] P. Lavoie, L. Djenidi, and R. A. Antonia. Effects of initial conditions in decaying turbulence generated by passive grids. J. Fluid Mech., 585:395-420, 2007. 